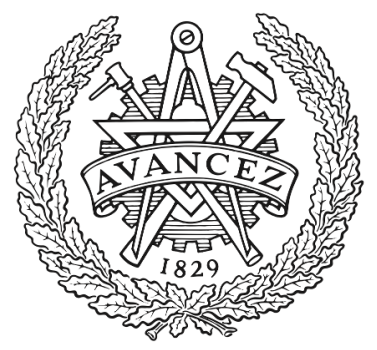

CHALMERS

UNIVERSITY OF TECHNOLOGY

\title{
Nondegenerate Parametric Resonance in a Tunable Superconducting Cavity
}

Downloaded from: https://research.chalmers.se, 2023-04-26 13:03 UTC

Citation for the original published paper (version of record):

Wustmann, W., Shumeiko, V. (2017). Nondegenerate Parametric Resonance in a Tunable

Superconducting Cavity. Physical Review Applied, 8(2).

http://dx.doi.org/10.1103/PhysRevApplied.8.024018

N.B. When citing this work, cite the original published paper. 


\title{
Nondegenerate Parametric Resonance in a Tunable Superconducting Cavity
}

\author{
Waltraut Wustmann ${ }^{1,2}$ and Vitaly Shumeiko ${ }^{1}$ \\ ${ }^{1}$ Chalmers University of Technology, S-41296 Göteborg, Sweden \\ ${ }^{2}$ Laboratory for Physical Sciences, College Park, Maryland 20740, USA \\ (Received 19 April 2017; published 21 August 2017)
}

\begin{abstract}
We develop a theory for nondegenerate parametric resonance in a tunable superconducting cavity. We focus on nonlinear effects that are caused by nonlinear Josephson elements connected to the cavity. We analyze parametric amplification in a strong nonlinear regime at the parametric-instability threshold, and we calculate maximum gain values. Above the threshold, in the parametric-oscillator regime, the cavity linear response diverges at the oscillator frequency at all pump strengths. We show that this divergence is related to the continuous degeneracy of the free oscillator state with respect to the phase. Applying on-resonance input lifts the degeneracy and removes the divergence. We also investigate quantum noise squeezing. It is shown that in the strong amplification regime, the noise undergoes four-mode squeezing, and that, in this regime, the output signal-to-noise ratio can significantly exceed the input value. We also analyze the intermode frequency conversion and identify the parameters at which full conversion is achieved.
\end{abstract}

DOI: $10.1103 /$ PhysRevApplied.8.024018

\section{INTRODUCTION}

Quantum parametric resonance in superconducting Josephson circuits finds numerous applications in circuitQED technology. A recently developed generation of quantum-limited parametric amplifiers [1-9] makes possible single-shot readout and the continuous monitoring of states of superconducting qubits [10-14]. Noise squeezing under parametric down-conversion [15] is used to enhance the qubit coherence time [16,17]. Among other applications are the efficient generation of entangled microwave photons [18-22] and intermode frequency conversion [23,24].

A detailed theory of degenerate parametric resonance in a tunable superconducting cavity was developed in Ref. [25]. In this paper, we extend this theory to the regime of nondegenerate resonance, when two cavity modes with frequencies $\omega_{n}$ and $\omega_{m}$ are coupled by parametric pumping with the frequency $\Omega \approx \omega_{n} \pm \omega_{m}$. A tunable superconducting cavity is a resonator integrated with a superconducting quantum-interferometer device (SQUID) that serves as a variable inductance controlled by magnetic flux [26,27]. Variation of the SQUID inductance changes the cavity resonance frequencies, and the parametric resonance is excited by rapid modulation of the SQUID inductance with an appropriate frequency (parametric flux pumping).

As was discussed in Ref. [25], a high-gain amplification regime in the vicinity of the parametric-instability threshold is strongly nonlinear. This nonlinearity limits the gain and squeezing at the threshold, and, moreover, it saturates the parametric instability and establishes a stationary oscillator regime above the threshold [28]. In contrast to optical parametric amplifiers and oscillators, where nonlinearity is typically related to pump depletion [29,30], the nonlinearity of the tunable cavity is introduced by the nonlinear inductance of the SQUID. In a small-amplitude limit, this is a cubic, Duffing-type nonlinearity of the Josephson current-phase dependence, which plays the role of the Kerr effect for the cavity field.

The nondegenerate parametric amplification possesses interesting features which are different from the degenerate case. Amplification of weak input follows a well-known two-mode-squeezing scenario of the linear parametricamplification theory [31-33]. However, the cavity linear response under the presence of a strong on-resonance intracavity field generated, e.g., by an amplified coherent signal or by the self-sustained parametric oscillation, exhibits four-mode squeezing. This effect is explained by the intracavity field acting as a secondary, "current" pump that excites additional idlers via four-mode mixing. As we show in this paper, the signal-to-noise ratio in this regime can be significantly enhanced compared to the input.

Furthermore, the cavity linear response in the parametric-oscillator regime diverges at the oscillation frequency at all pump strengths. This phenomenon is analogous to the one in the optical parametric oscillators, which has attracted a great deal of attention [29,34-36] (for more-recent discussions see, e.g., Refs. [37,38]). This divergence is closely related to the continuous degeneracy of the free oscillator state with respect to the oscillation phase, and it is lifted by applying an on-resonance input.

The structure of the paper is as follows. In Sec. II, we briefly outline a description of the tunable cavity developed in Ref. [25], and we introduce dynamical equations for the nondegenerate parametric resonance. Section III is devoted to the parametric-amplification regime. First, we consider parametric instability and parametric oscillation. Then we proceed with a classical theory of nonlinear amplification across the parametric threshold. Sections III D and III E are devoted to the detailed study of the linear response of the 
empty cavity, relevant for the noise squeezing, and the multimode response of the filled cavity using the framework of supermodes $[39,40]$. We discuss the effect of continuous degeneracy of the parametric oscillation and phase locking by means of weak signal injection. In Sec. IV, we study quantum noise squeezing; we compute quadrature correlation functions and analyze the signal-to-noise ratio for linear and nonlinear amplification regimes. At the end of the section, we present explicit equations for a squeezed vacuum below the parametric threshold. In Sec. V, we derive the scattering matrix for the parametric frequency conversion.

\section{TUNABLE CAVITY}

The tunable cavity we study in this paper is a $\lambda / 4$ superconducting resonator connected at one end to a SQUID, and at the other end to a transmission line; see Fig. 1. The parametric effect is achieved by the rapid temporal modulation of a magnetic flux through the SQUID, which results in a variation of the boundary condition at the cavity edge that shifts the cavity resonance frequencies.

In this paper, we consider the nondegenerate parametric resonance, which corresponds to the modulation with frequency close to the sum, or difference, of the cavity eigenmode frequencies, $\Omega \approx \omega_{2} \pm \omega_{1}$. Our present study is built on a detailed analysis of this device in the context of the degenerate parametric regime [25]. Here, we briefly outline the results of that analysis.

The quantum Hamiltonian of the tunable cavity derived in Ref. [25] has the form

$$
\begin{aligned}
H_{\mathrm{cav}}\left(a_{n}\right) & =\sum_{n} \hbar \omega_{n} a_{n}^{\dagger} a_{n}+V\left(a_{n}, t\right), \\
V\left(a_{n}, t\right) & =-\left(E_{J} \sin F\right)(\delta f \cos \Omega t) \phi_{d}^{2}-\frac{E_{J} \cos F}{12} \phi_{d}^{4} .
\end{aligned}
$$

This Hamiltonian describes the field inside the cavity, $\phi(x, t)$, in terms of the cavity eigenmodes,

$$
\phi(x, t)=\sqrt{\frac{(2 e)^{2}}{\hbar C_{\mathrm{cav}}}} \sum_{n=1}^{\infty} \frac{\cos \left(k_{n} x\right)}{\sqrt{\omega_{n}}}\left[a_{n}(t)+a_{n}^{\dagger}(t)\right] .
$$

In these equations, $E_{J}$ is the Josephson energy of the SQUID junction, $F$ is the constant magnetic flux bias; $\delta f$ is the amplitude of the flux temporal modulation; $\phi_{d}(t)$ refers

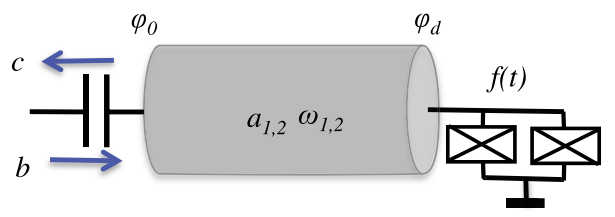

FIG. 1. Sketch of tunable cavity. $a_{n}(t)$ is the complex amplitude of the $n$th cavity eigenmode, $b(t)$ and $c(t)$ are input and output field amplitudes, respectively; the cavity eigenfrequencies $\omega_{n}$ are controlled by the magnetic flux $f(t)=F+\delta f \cos \Omega t$. to the field boundary value at the cavity edge, $x=d$, connected to the SQUID; $C_{\text {cav }}$ is the cavity capacitance; $k_{n}=\omega_{n} / v$ is the mode eigenvector; $v=d / \sqrt{C_{\text {cav }} L_{\text {cav }}}$ is the electromagnetic-wave velocity; $a_{n}(t)$ represents the mode annihilation operators, which satisfy the bosonic commutation relations, $\left[a_{n}, a_{m}^{\dagger}\right]=\delta_{n m}$.

The cavity mode spectrum is defined by the equation

$$
\left(k_{n} d\right) \tan k_{n} d=\frac{2 E_{J} \cos F}{E_{L, \mathrm{cav}}}=\frac{1}{\gamma},
$$

where $\gamma$ is a participation ratio of the inductive energies of the cavity, $E_{L \text {, cav }}=(\hbar / 2 e)^{2}\left(1 / L_{\text {cav }}\right)$, and the SQUID. The participation ratio is small, $\gamma \ll 1$, for magnetic flux bias not too close to $\pi / 2$. A weak effect of the capacitance of the Josephson junction, which is small compared to the cavity capacitance $C_{\text {cav }}$, is neglected here. The nonequidistant character of the cavity spectrum allows the selective parametric excitation of only two cavity modes.

Important constraints under which the Hamiltonian (1) is valid concern small values of the amplitudes of the parametric modulation and the field at the cavity edge,

$$
\delta f, \phi_{d} \ll 1 .
$$

\section{A. Equation of motion}

The quantum dynamics of the field in the cavity is described by the set of Langevin equations associated with the Hamiltonian (1),

$i \dot{a}_{n}-\omega_{n} a_{n}-\frac{1}{\hbar}\left[a_{n}, V\left(a_{n}, t\right)\right]+i \Gamma_{n} a_{n}=\sqrt{2 \Gamma_{n 0}} b(t)$.

These equations take into account external losses related to the coupling to the transmission line [25],

$$
\Gamma_{n 0}=\omega_{n}\left(\frac{C_{c}}{C_{\text {cav }}}\right)^{2} k_{n} d
$$

where $C_{c}$ is the coupling capacitance and $\Gamma_{n}$ refers to the total losses. The operator $b(t)$ indicates an input field defined through the mode operators of the transmission line, $a_{k}(t)[41,42]$,

$$
b(t)=\sqrt{\frac{v}{2 \pi}} \int_{0}^{\infty} d k a_{k}\left(t_{0}\right) e^{-i \omega_{k}\left(t-t_{0}\right)} .
$$

The output field operator $c_{n}(t)$ is related to the input operator via the relation [41]

$$
c_{n}(t)=b(t)-i \sqrt{2 \Gamma_{n 0}} a_{n}(t) .
$$

\section{B. Resonance approximation}

The nondegenerate parametric resonance has two qualitatively different regimes. The first, the amplification or 
down-conversion regime, corresponds to the pump frequency close to the sum of the frequencies of the two selected modes,

$$
\Omega=\omega_{2}+\omega_{1}+2 \delta, \quad \delta \ll \omega_{n} .
$$

For the amplification regime, a natural reference frame is a rotating frame with frequencies $\omega_{1,2}+\delta$. In this frame, the mode operators undergo the transformations

$$
a_{n} \rightarrow e^{-i\left(\omega_{n}+\delta\right) t} a_{n}
$$

The corresponding transformation of the cavity Hamiltonian is produced by a unitary operator,

$$
\begin{aligned}
& U=\exp \left[-i\left(\omega_{1}+\delta\right) a_{1}^{\dagger} a_{1}-i\left(\omega_{2}+\delta\right) a_{2}^{\dagger} a_{2}\right], \\
& H_{\mathrm{cav}} \rightarrow H_{\mathrm{cav}}-i \hbar \dot{U} U^{\dagger}=H_{\mathrm{cav}}-\hbar \sum_{n}\left(\omega_{n}+\delta\right) a_{n}^{\dagger} a_{n} .
\end{aligned}
$$

Averaging over rapid oscillations yields the reduced Hamiltonian of the resonance approximation,

$$
\begin{aligned}
H_{\mathrm{cav}}= & -\sum_{n=1,2}\left[\hbar \delta a_{n}^{\dagger} a_{n}+\frac{\hbar \alpha_{n}}{2}\left(a_{n}^{\dagger} a_{n}+\frac{1}{2}\right)^{2}\right] \\
& -2 \hbar \alpha\left(a_{1}^{\dagger} a_{1}+\frac{1}{2}\right)\left(a_{2}^{\dagger} a_{2}+\frac{1}{2}\right)-\hbar \epsilon\left(a_{1} a_{2}+a_{1}^{\dagger} a_{2}^{\dagger}\right) .
\end{aligned}
$$

Here, we define the mode-specific nonlinearity parameters,

$$
\begin{aligned}
\alpha_{n} & =\frac{\hbar}{2 \gamma E_{L, \mathrm{cav}}}\left(\frac{\sqrt{\omega_{n}} \cos k_{n} d}{k_{n} d}\right)^{4}, \\
\alpha & =\sqrt{\alpha_{1} \alpha_{2}},
\end{aligned}
$$

and a pump strength,

$$
\epsilon=\frac{\delta f \tan F}{2 \gamma}\left(\frac{\sqrt{\omega_{1}} \cos k_{1} d}{k_{1} d}\right)\left(\frac{\sqrt{\omega_{2}} \cos k_{2} d}{k_{2} d}\right) .
$$

The resonance approximation for the Langevin equations yields two coupled equations,

$$
\begin{aligned}
& i \dot{a}_{n}+\delta a_{n}+i \Gamma_{n} a_{n}+\epsilon a_{m}^{\dagger}+\alpha_{n}\left(a_{n}^{\dagger} a_{n}+1\right) a_{n} \\
& \quad+2 \alpha\left(a_{m}^{\dagger} a_{m}+\frac{1}{2}\right) a_{n}=\sqrt{2 \Gamma_{n 0}} b_{n}(t),
\end{aligned}
$$

where $m \neq n$, and the input fields, $b_{n}(t)$, are written in the respective rotating frames, $\omega_{n}+\delta$. The input-output relations in Eq. (8) retain their form in the rotating frame.

The alternative regime of parametric frequency conversion or up-conversion corresponds to the pump frequency close to the difference of the frequencies of two selected modes,

$$
\Omega=\omega_{2}-\omega_{1}+2 \delta, \quad \omega_{2}>\omega_{1} .
$$

In this case, no amplification occurs while the resonant modes are hybridized, and the energy is transferred from one mode to the other. For this regime, a natural reference frame is a rotating frame with the frequencies $\omega_{1}-\delta$ and $\omega_{2}+\delta$, i.e.,

$$
a_{1} \rightarrow e^{-i\left(\omega_{1}-\delta\right) t} a_{1}, \quad a_{2} \rightarrow e^{-i\left(\omega_{2}+\delta\right) t} a_{2} .
$$

The corresponding unitary operator is

$$
U=\exp \left[-i\left(\omega_{1}-\delta\right) a_{1}^{\dagger} a_{1}-i\left(\omega_{2}+\delta\right) a_{2}^{\dagger} a_{2}\right] .
$$

The resonant Langevin equations in the rotating frame take the form

$$
\begin{gathered}
i \dot{a}_{n} \mp \delta a_{n}+i \Gamma_{n} a_{n}+\epsilon a_{m}+\alpha_{n}\left(a_{n}^{\dagger} a_{n}+1\right) a_{n} \\
\quad+2 \alpha\left(a_{m}^{\dagger} a_{m}+\frac{1}{2}\right) a_{n}=\sqrt{2 \Gamma_{n 0}} b_{n}(t),
\end{gathered}
$$

where the upper and lower signs refer to modes 1 and 2 , respectively.

The resonance approximation implies a slow resonance dynamics on the time scale set by the cavity eigenfrequencies,

$$
\delta, \epsilon, \alpha_{n}\left|A_{n}\right|^{2}, \Gamma_{n} \ll \omega_{n}
$$

(here, $A_{n}$ indicates a quasiclassical intracavity field). Bearing in mind that, for a small $\gamma$, the estimate, $\cos k_{n} d \sim$ $\gamma \ll 1$, holds for low-frequency modes and a bias flux $F$ not particularly close to $\pi / 2$, and using Eqs. (2), (15), and (13), we are able to obtain the relations

$$
\frac{\epsilon}{\omega_{n}} \sim \gamma \delta f \tan F \ll 1, \quad \frac{\alpha_{n}\left|A_{n}\right|^{2}}{\omega_{n}} \sim \gamma \phi_{d}^{2} \ll 1 .
$$

These inequalities respect constraints in Eq. (21), and they are automatically fulfilled by virtue of the constraints in Eq. (4). Furthermore, the relations in Eq. (21) provide room for validity of the theory well above the parametricoscillation threshold for a high-quality cavity,

$$
\begin{aligned}
& \Gamma_{n} \ll \epsilon \sim \omega_{n} \gamma \delta f \tan F \ll \omega_{n}, \\
& \Gamma_{n} \ll \alpha_{n}\left|A_{n}\right|^{2} \sim \omega_{n} \gamma \phi_{d}^{2} \ll \omega_{n} .
\end{aligned}
$$

\section{PARAMETRIC AMPLIFICATION}

We start our study with the classical amplification regime. By denoting classical fields with capital letters, $A_{n}$, we write a classical version of Eq. (16) in the form 


$$
\begin{aligned}
i \dot{A}_{1}+\left(\zeta_{1}+i \Gamma_{1}\right) A_{1}+\epsilon A_{2}^{*} & =\sqrt{2 \Gamma_{10}} B_{1}(t), \\
-i \dot{A}_{2}^{*}+\left(\zeta_{2}-i \Gamma_{2}\right) A_{2}^{*}+\epsilon A_{1} & =\sqrt{2 \Gamma_{20}} B_{2}^{*}(t),
\end{aligned}
$$

where

$$
\begin{aligned}
& \zeta_{1}=\delta+\alpha_{1}\left|A_{1}\right|^{2}+2 \alpha\left|A_{2}\right|^{2}, \\
& \zeta_{2}=\delta+\alpha_{2}\left|A_{2}\right|^{2}+2 \alpha\left|A_{1}\right|^{2} .
\end{aligned}
$$

These terms describe the nonlinear self-Kerr effect proportional to $\alpha_{n}$, and the cross-Kerr effect proportional to $\alpha$.

\section{A. Parametric instability and oscillation}

First, we consider the dynamics of the closed cavity. The empty cavity state, $A_{n}=0$, always exists but loses stability within a certain region of the pump strengths and detunings. To perform the stability analysis, we evaluate the eigenfrequency spectrum of the linearized equation (24). Assuming $\zeta_{n}=\delta$ and $A_{1}(t), A_{2}^{*}(t) \propto e^{-i \Delta t}$, we compute the determinant of the dynamical matrix,

$$
\text { Det }=\left(\Delta+\delta+i \Gamma_{1}\right)\left(-\Delta+\delta-i \Gamma_{2}\right)-\epsilon^{2}=0 .
$$

Within the stability region, the solution to this equation must have a negative imaginary part, $\operatorname{Im} \Delta \leq 0$, and the condition, $\operatorname{Im} \Delta=0$, must therefore define the boundary of this region, i.e., the instability threshold. Using this argument, we solve Eq. (26) separately for the real and imaginary parts and find the threshold,

$$
\begin{aligned}
& \epsilon^{2}=\Gamma_{1} \Gamma_{2}+\delta^{2}\left[1-\left(\frac{\Gamma_{1}-\Gamma_{2}}{\Gamma_{1}+\Gamma_{2}}\right)^{2}\right], \\
& \Delta=\frac{\Gamma_{1}-\Gamma_{2}}{\Gamma_{1}+\Gamma_{2}} \delta .
\end{aligned}
$$

As one can see in these equations, the instability occurs at frequencies generally deviating from the cavity resonances. The instability threshold has a minimum value at the zero pump detuning $\delta$, where it is defined by the damping, $\epsilon=\sqrt{\Gamma_{1} \Gamma_{2}}$; the threshold grows with the pump detuning, as illustrated in Fig. 2(a).

In terms of the pump detuning, the cavity ground state is unstable within the interval,

$$
\delta^{2}<\delta_{\mathrm{th}}^{2}=\frac{\left(\Gamma_{1}+\Gamma_{2}\right)^{2}}{4 \Gamma_{1} \Gamma_{2}}\left(\epsilon^{2}-\Gamma_{1} \Gamma_{2}\right) .
$$

For modes with equal dampings, $\Gamma_{1}=\Gamma_{2}$, the threshold value becomes identical to the one for the degenerate case [25], and the deviation of the critical-fluctuation frequency turns to zero, $\Delta=0$.

The instability leads to the emergence of a self-sustained parametric oscillation above the threshold, and it is described with a nonlinear solution of the homogeneous
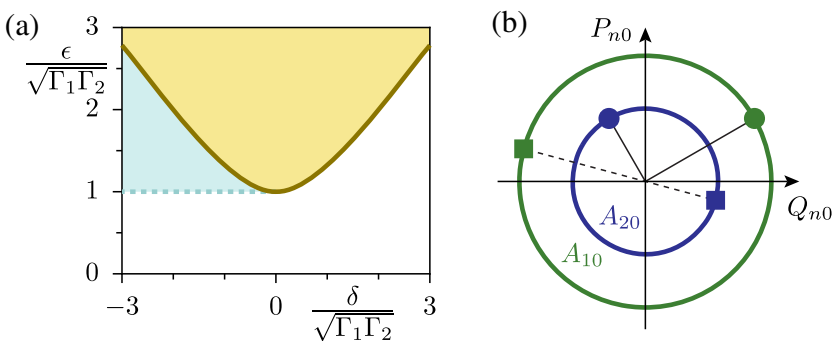

FIG. 2. (a) Region of parametric oscillation in the $(\epsilon-\delta)$ plane (yellow), and the coexistence region of parametric oscillation and empty cavity states (blue). (b) Quadratures $Q_{n 0}=\left(A_{n 0}+A_{n 0}^{*}\right) / 2$ and $P_{n 0}=\left(A_{n 0}-A_{n 0}^{*}\right) /(2 i)$ of a parametric-oscillation state forming circles, with radii determined by Eqs. (31) and (32), and phases constrained by Eq. (34); indicated are the state pairs $\left(Q_{n}, P_{n}\right)$ for two specific values of the phase difference, $\psi=$ $-\pi / 2$ (the circles) and $\psi=\pi$ (the squares), the phase sum $\Theta=$ $5 \pi / 6$ remains invariant. $\epsilon=2 \sqrt{\Gamma_{1} \Gamma_{2}}, \delta=0, \Gamma_{2}=3 \Gamma_{1}$, and $\alpha_{2}=3 \alpha_{1}=3 \sqrt{\Gamma_{1} \Gamma_{2}} / 100$.

equation (24). This regime is manifested by spontaneous radiation from the cavity at two frequencies shifted from the cavity resonances, $\omega_{1}+\delta+\Delta_{0}$ and $\omega_{2}+\delta-\Delta_{0}$. This characteristics is different from the degenerate case, where the parametric radiation frequency coincides with half of the pump frequency, $\Omega / 2$, and coincides with the cavity mode frequency at zero pump detuning, $\delta=0$.

The oscillation frequency shift is derived in the Appendix,

$$
\Delta_{0}=\frac{\Gamma_{1} \zeta_{2}-\Gamma_{2} \zeta_{1}}{\Gamma_{1}+\Gamma_{2}}
$$

It grows with increasing pump intensity and vanishes only if the modes have identical characteristics, $\Gamma_{1}=\Gamma_{2}$ and $\alpha_{1}=\alpha_{2}$. Following the terminology of Ref. [34], we will call such modes balanced.

The oscillation is characterized by complex amplitudes, $A_{n 0}=\left|A_{n 0}\right| e^{i \theta_{n}}$, whose moduli are related,

$$
\frac{\left|A_{20}\right|^{2}}{\left|A_{10}\right|^{2}}=\frac{\Gamma_{1}}{\Gamma_{2}}
$$

as is found in the Appendix, and

$$
\left|A_{10}\right|^{2}=\frac{2\left(-\delta \mp \delta_{\mathrm{th}}\right) \Gamma_{2}}{\alpha_{1} \Gamma_{2}+\alpha_{2} \Gamma_{1}+2 \alpha\left(\Gamma_{1}+\Gamma_{2}\right)} .
$$

Similar to the degenerate case, Eq. (32) describes unstable (the upper sign) and stable (the lower sign) oscillator states. The stable state exists at all $\delta<\delta_{\text {th }}$, and it coexists with the stable trivial state at $\delta<-\delta_{\text {th }}$ [see Fig. 2(a)]; in the latter region, both the excited and trivial stable states are separated by an unstable state. For the balanced modes, Eq. (32) reduces to the one for a degenerate oscillator [Eq. (45) in Ref. [25]], 


$$
\left|A_{0}\right|^{2}=\frac{-\delta \mp \sqrt{\epsilon^{2}-\Gamma^{2}}}{3 \alpha}
$$

with rescaling, $\alpha \rightarrow 3 \alpha$, that stems from the cross-Kerr effect.

The properties of the oscillation phases $\theta_{n}$ are qualitatively different from the degenerate case: there, the phase takes two values differing by $\pi$, implying double degeneracy of the oscillator state. Here, the oscillator state has a continuous degeneracy with respect to the difference, $\psi=\theta_{1}-\theta_{2}$, of the mode phases. The sum of the phases is fixed and, for the stable state, reads, according to Eqs. (A3) and (A8),

$$
\begin{aligned}
\sin \Theta & =\frac{\sqrt{\Gamma_{1} \Gamma_{2}}}{\epsilon}, \quad \cos \Theta=-\frac{\sqrt{\epsilon^{2}-\Gamma_{1} \Gamma_{2}}}{\epsilon}, \\
\Theta & =\theta_{1}+\theta_{2} \in(\pi / 2, \pi) .
\end{aligned}
$$

The output radiation is connected to the intracavity field via the relation

$$
C_{n 0}=\left|C_{n 0}\right| e^{i \theta_{C n}}=-i \sqrt{2 \Gamma_{n 0}} A_{n 0},
$$

and the output radiation phases are then related,

$$
\theta_{C 1}+\theta_{C 2}=\Theta-\pi
$$

as are the radiation intensities,

$$
\frac{\left|C_{20}\right|^{2}}{\left|C_{10}\right|^{2}}=\frac{\Gamma_{1} \Gamma_{20}}{\Gamma_{2} \Gamma_{10}}
$$

In the ideal cavity, $\Gamma_{n}=\Gamma_{n 0}$, the radiation intensities are equal in both modes.

\section{B. Nonlinear gains}

Now we switch on an input in Eq. (24) and suppose a harmonic input in the first mode, $B_{1}(t)=B_{1}(\Delta) e^{-i \Delta t}$, slightly detuned, by $\Delta$, from the reference frame, $\omega_{1}+\delta$, and having a complex amplitude, $B_{1}(\Delta)$. Below the parametric threshold, this signal would generate an intracavity field consisting of two harmonics, the signal, $A_{1}(t)=$ $A_{1}(\Delta) e^{-i \Delta t}$, and the idler, $A_{2}(t)=A_{2}(-\Delta) e^{i \Delta t}$. The idler is detuned by $-\Delta$ from its reference frequency, $\omega_{2}+\delta$. The same harmonic components will be present in the output field, $C_{1}(t)=C_{1}(\Delta) e^{-i \Delta t}$ and $C_{2}(t)=C_{2}(-\Delta) e^{i \Delta t}$.

This arrangement may also include a second input signal at the idler frequency, $B_{2}(t)=B_{2}(-\Delta) e^{i \Delta t}$. Then Eq. (24) reduces to a static equation for intracavity-field amplitudes,

$$
\begin{aligned}
\left(\Delta+\zeta_{1}+i \Gamma_{1}\right) A_{1}(\Delta)+\epsilon A_{2}^{*}(-\Delta) & =\sqrt{2 \Gamma_{10}} B_{1}(\Delta), \\
\left(-\Delta+\zeta_{2}-i \Gamma_{2}\right) A_{2}^{*}(-\Delta)+\epsilon A_{1}(\Delta) & =\sqrt{2 \Gamma_{20}} B_{2}^{*}(-\Delta) .
\end{aligned}
$$

Inverting these equations yields explicitly the intracavity field,

$$
\left(\begin{array}{c}
A_{1}(\Delta) \\
A_{2}^{*}(-\Delta)
\end{array}\right)=\mathcal{A}(\Delta)\left(\begin{array}{c}
\sqrt{2 \Gamma_{10}} B_{1}(\Delta) \\
\sqrt{2 \Gamma_{20}} B_{2}^{*}(-\Delta)
\end{array}\right),
$$

where

$$
\begin{aligned}
\mathcal{A}(\Delta) & =\frac{1}{\operatorname{Det}}\left(\begin{array}{cc}
-\Delta+\zeta_{2}-i \Gamma_{2} & -\epsilon \\
-\epsilon & \Delta+\zeta_{1}+i \Gamma_{1}
\end{array}\right), \\
\operatorname{Det}(\Delta) & =\left(\Delta+\zeta_{1}+i \Gamma_{1}\right)\left(-\Delta+\zeta_{2}-i \Gamma_{2}\right)-\epsilon^{2} .
\end{aligned}
$$

This formal solution for the intracavity field, together with Eq. (8), allows us to formulate the input-output relation, the Bogoliubov transformation,

$$
\left(\begin{array}{c}
C_{1}(\Delta) \\
C_{2}^{*}(-\Delta)
\end{array}\right)=\mathcal{V}(\Delta)\left(\begin{array}{c}
B_{1}(\Delta) \\
B_{2}^{*}(-\Delta)
\end{array}\right)
$$

The input-output matrix elements are

$$
\begin{aligned}
& \mathcal{V}_{11}(\Delta)=1-\frac{2 i \Gamma_{10}\left(-\Delta-i \Gamma_{2}+\zeta_{2}\right)}{\operatorname{Det}(\Delta)}, \\
& \mathcal{V}_{22}(\Delta)=1+\frac{2 i \Gamma_{20}\left(\Delta+i \Gamma_{1}+\zeta_{1}\right)}{\operatorname{Det}(\Delta)} \\
& \mathcal{V}_{12}(\Delta)=\frac{2 i \epsilon \sqrt{\Gamma_{10} \Gamma_{20}}}{\operatorname{Det}(\Delta)}=-\mathcal{V}_{21}(\Delta) .
\end{aligned}
$$

One can straightforwardly verify that matrix $\mathcal{V}(\Delta)$ possesses the properties

$$
\left|\mathcal{V}_{11}\right|^{2}-\left|\mathcal{V}_{12}\right|^{2}=1, \quad\left|\mathcal{V}_{22}\right|=\left|\mathcal{V}_{11}\right|, \quad\left|\mathcal{V}_{12}\right|=\left|\mathcal{V}_{21}\right|,
$$

in the absence of internal losses, $\Gamma_{n}=\Gamma_{n 0}$.

Amplification of a single-mode input, $B_{1}(\Delta)$, is characterized by the gains

$$
\begin{gathered}
G_{11}(\Delta)=\left|\frac{C_{1}(\Delta)}{B_{1}(\Delta)}\right|^{2}=\left|\mathcal{V}_{11}(\Delta)\right|^{2}, \\
G_{12}(-\Delta)=\left|\frac{C_{2}(-\Delta)}{B_{1}(\Delta)}\right|^{2}=\left|\mathcal{V}_{21}(\Delta)\right|^{2} .
\end{gathered}
$$

These gains are nonlinear functions of the input due to dependence on the intracavity field entering the Kerr terms, $\zeta_{n}\left(A_{1}, A_{2}\right)$ in Eq. (42). Nevertheless, the nonlinear gains respect the relations

$$
\begin{aligned}
& G_{11}(\Delta)=1+G_{12}(-\Delta), \\
& G_{11}(\Delta)=G_{22}(-\Delta), \quad G_{21}(\Delta)=G_{12}(-\Delta),
\end{aligned}
$$

following from Eq. (43), which are the same as the ones known from the theory of linear amplifiers $[31,33]$.

As we see later in this section, the cavity response becomes increasingly nonlinear while approaching the 
instability threshold, where amplification of even a singlephoton input becomes strongly nonlinear.

The application of the nonlinear equation (38) above the parametric threshold requires certain care. In this region, the stationary response is possible only for an input whose frequency coincides with the frequency of parametric oscillation, $\Delta=\Delta_{0}$. For detuned inputs, the nonlinear response is nonstationary due to the mixing of the input and oscillation fields by the Kerr effect. If the input is so weak that its contribution to the nonlinear terms can be neglected, the linearized response is stationary.

\section{Amplification of on-resonance signal}

In this section, we analyze the nonlinear amplification of a single on-resonance input signal to get a general picture of the nonlinear amplification during transition through the parametric-oscillation threshold. The results in this section are presented for relatively weak input power, $\left|B_{1}\right|^{2} \lesssim \sqrt{\Gamma_{1} \Gamma_{2}}$, and neglecting intrinsic losses, $\Gamma_{n}=\Gamma_{n 0}$.

\section{Balanced modes}

We first investigate numerically a simpler case of balanced modes $\left(\alpha_{n}=\alpha\right.$ and $\left.\Gamma_{n}=\Gamma\right)$. In this case, the parametric oscillation has zero detuning, $\Delta_{0}=0$, and we explore amplification of the on-resonance signal with $\Delta=0$, both below and above the threshold.

In Fig. 3, the computed cavity responses of both modes, $\left|C_{1,2}\right|^{2}$, are presented as functions of the pump detuning $\delta$ below and above the parametric threshold. The characteristic responses of both modes are qualitatively similar and resemble the response of the degenerate parametric amplifier (cf. Fig. 5 and the discussion in Ref. [25]).

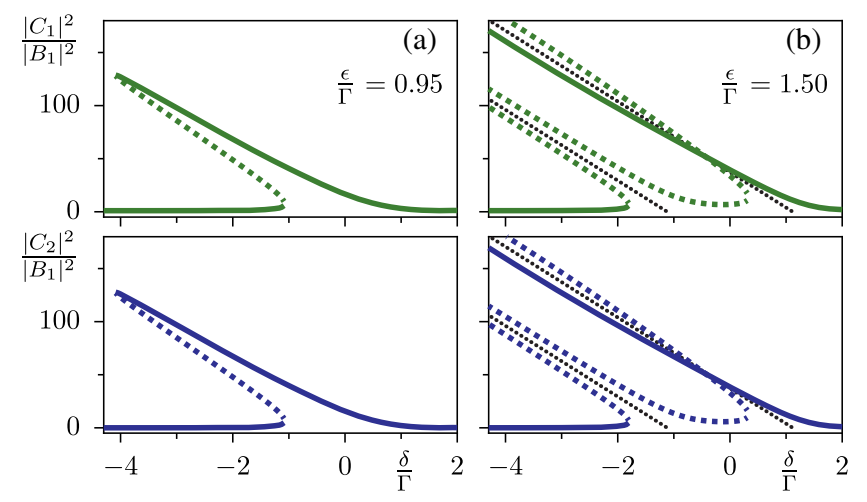

FIG. 3. Nonlinear gains for signal and idler $\left|C_{1,2}\right|^{2}$ (the upper and lower panels, respectively) for the undetuned, $\Delta=0$ input signal $B_{1}$, following from Eqs. (39) and (8) for balanced modes, $\Gamma_{1}=\Gamma_{2}$, and $\alpha_{1}=\alpha_{2}$, as functions of pump detuning $\delta$ for different values of the pump strength $\epsilon$ : (a) below threshold, $\epsilon / \Gamma=0.95$, and (b) above threshold, $\epsilon / \Gamma=1.5$. The solid (dashed) lines indicate stable (unstable) states; thin dotted lines indicate radiation amplitudes from stable and unstable parametric-oscillator states. $\left|B_{1}\right|^{2}=2 \Gamma, \theta_{B}=0, \alpha=\Gamma / 100$, and $\Gamma=\Gamma_{0}$.
For an $\epsilon$ smaller than the minimum parametric threshold, $\epsilon<\Gamma$, the response curves $\left|C_{1,2}\right|^{2}(\delta)$ in Fig. 3(a) essentially describe a Duffing resonance. When approaching the threshold, $\epsilon \lesssim \Gamma$, the maxima of $\left|C_{1,2}\right|^{2}(\delta)$ are strongly enhanced, while the resonance width decreases owing to the reduced effective damping, $\Gamma^{2} \rightarrow \Gamma^{2}-\epsilon^{2}$.

The similarity to the degenerate resonance case is, however, illusive and does not reflect the fact that the intracavity dynamics here occurs in a higher-dimensional phase space and is more complicated. We attribute this similarity to the fact that the cavity steady state here remains close to the manifold characterized by $\left|A_{1}\right|=\left|A_{2}\right|$, which amounts to a projection to the degenerate subspace. This is a rather special situation which stems from the mode symmetry.

When $\epsilon>\Gamma$, Fig. 3(b), the resonance splits into two branches, with each branch consisting of a pair of steady states. The lower-amplitude pair is formed close to the amplitude of the unstable parametric-oscillation state (the dotted line), and both states of this pair are unstable as well. The higher-amplitude pair is formed close to the amplitude of the stable parametric-oscillation state (the dotted line), but only one of its two components is stable. This stability characteristics is in contrast to the degenerate parametric amplifier, where both components of the higher-amplitude pair are stable, [Figs. 5(c) and 5(d) in Ref. [25]].

The difference can be understood from the underlying parametric-oscillation states, from which the branches emerge: the degenerate parametric oscillator has two degenerate stable oscillation states which are shifted by $\pi$ in phase. When a small external signal is applied, each of these states remains stable, shifting only in the quadrature plane. By contrast, the nondegenerate parametric oscillator has infinitely many stable oscillation states which are degenerate with respect to both $\left|A_{1}\right|^{2}$ and $\left|A_{2}\right|^{2}$, but they differ by an arbitrary phase difference $\theta_{1}-\theta_{2}$. A small external signal breaks this rotational symmetry since $\theta_{1}-\theta_{2}$ acquires a fixed, $B$-dependent value according to Eq. (39) [see Eq. (55) below, and further analytical details in Sec. III F 1].

\section{Unbalanced modes}

In this section, we consider the more realistic case of unbalanced modes with nonidentical parameters. The mode parameters are specified by Eqs. (6) and (13), and in the limit $\gamma \ll 1$, they have the scaling

$$
\frac{\Gamma_{n 0}}{\Gamma_{m 0}}=\frac{\alpha_{n}}{\alpha_{m}} \approx\left(\frac{2 n-1}{2 m-1}\right)^{2}
$$

To explore this more complex case, we have to take into account the nonzero detuning of the parametricoscillator frequency $\Delta_{0}$, and we therefore consider input that is on resonance with the oscillator, $\Delta=\Delta_{0}(\delta)$, above 
the threshold, while keeping (for consistency) the finite detuning, $\Delta=\Delta_{0}\left(\delta_{\text {th }}\right)$, below the threshold.

The most important implication of the mode asymmetry is that the internal cavity dynamics is no longer confined to the vicinity of the manifold $\left|A_{1}\right|=\left|A_{2}\right|$ in phase space. This increased phase space dimension has consequences for the cavity response already below threshold, as demonstrated in Fig. 4(a). Here, the effectively reduced damping at strong pumping, $\epsilon^{2} \sim \Gamma_{1} \Gamma_{2}$ in Eq. (40), does not result in a strongly enhanced Duffing resonance. Therefore, the cavity does not automatically enter a regime of multistability, as it does in the case of the degenerate parametric amplifier at small input. This behavior is related to the fact that the internal cavity amplitudes $\left|A_{1,2}\right|^{2}$ differ strongly, although the illustrated output amplitudes $\left|C_{1,2}\right|^{2}$ are practically identical.

Above threshold, Fig. 4(b), we still observe a stable highamplitude cavity state extending far at red detuning, $\delta<\delta_{\text {th }}$, but the unstable branch is confined to a small window of the red-detuned $\delta$, Fig. 4(b).

\section{Nonlinear amplification close to threshold}

Some analytical results can be obtained for the regime of strong nonlinear amplification in close proximity to the threshold. In this regime, the width of the resonance is determined by the nonlinear frequency shifts, $\alpha_{n}\left|A_{n}\right|^{2}$, while these terms can still be relatively small,

$$
\left|1-\frac{\epsilon^{2}}{\Gamma_{1} \Gamma_{2}}\right| \ll \frac{\alpha_{n}\left|A_{n}\right|^{2}}{\Gamma_{n}} \ll 1 .
$$

This regime starts below the threshold, and it persists across the threshold as long as the contribution of the input

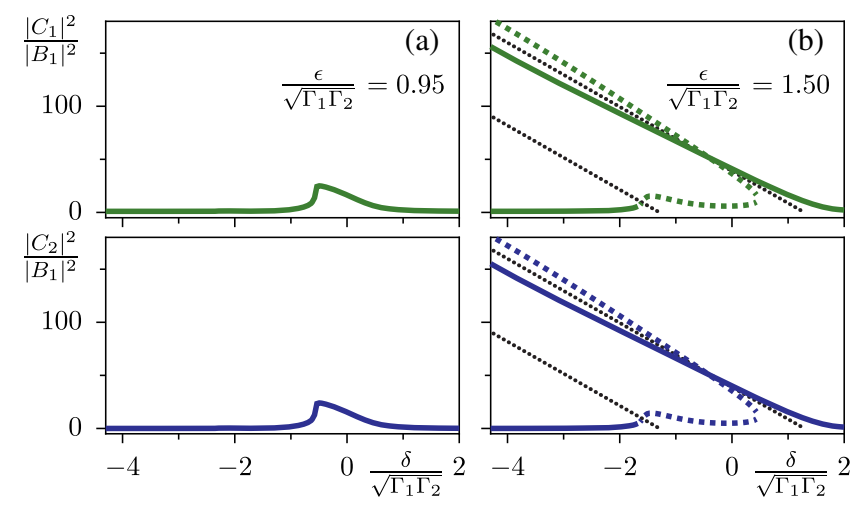

FIG. 4. Nonlinear gains for signal and idler, $\left|C_{1,2}\right|^{2}$ (the upper and lower panels, respectively), for the unbalanced modes $\Gamma_{2}=3 \Gamma_{1}$ and $\alpha_{2}=3 \alpha_{1}$, as functions of pump detuning $\delta$ for different values of the pump strength $\epsilon$ : (a) below threshold, $\epsilon / \sqrt{\Gamma_{1} \Gamma_{2}}=0.95$, and (b) above threshold, $\epsilon / \sqrt{\Gamma_{1} \Gamma_{2}}=1.5$. In (b), the input is detuned, $\Delta=\Delta_{0}(\delta)$, to be on resonance with the parametric radiation. The thin dotted lines indicate radiation amplitudes from stable and unstable parametric-oscillator states. $\left|B_{1}\right|^{2}=2 \sqrt{\Gamma_{1} \Gamma_{2}}, \theta_{B}=0, \alpha_{1}=\sqrt{\Gamma_{1} \Gamma_{2}} / 100$, and $\Gamma_{n}=\Gamma_{n 0}$. to the Kerr terms dominates over that of the emerging parametric oscillation. In principle, this regime is relevant for any value of pump and input detunings, but we focus, for the sake of simplicity, on the case $\delta=\Delta=0$ and neglect the internal losses, $\Gamma_{n}=\Gamma_{n 0}$.

Then the solution for the intracavity field, Eq. (40), takes an approximate form [for a single input $B_{1}(0)$ ],

$$
\begin{aligned}
A_{1} & =\frac{-i \Gamma_{2}}{\operatorname{Det}} \sqrt{2 \Gamma_{1}} B_{1}, \quad A_{2}^{*}=\frac{-\epsilon}{\operatorname{Det}} \sqrt{2 \Gamma_{1}} B_{1}, \\
\operatorname{Det} & =\zeta_{1} \zeta_{2}+i\left(\Gamma_{1} \zeta_{2}-\Gamma_{2} \zeta_{1}\right) .
\end{aligned}
$$

From these equations, we deduce

$$
\left|\frac{A_{2}}{A_{1}}\right|^{2}=\frac{\epsilon^{2}}{\Gamma_{2}^{2}} \approx \frac{\Gamma_{1}}{\Gamma_{2}} .
$$

Using this relation, we compute the second term in the determinant in Eq. (48),

$$
i\left(\Gamma_{1} \zeta_{2}-\Gamma_{2} \zeta_{1}\right)=i \frac{\left|A_{1}\right|^{2}}{\Gamma_{2}}\left(\alpha_{2} \Gamma_{1}^{2}-\alpha_{1} \Gamma_{2}^{2}\right)
$$

For unbalanced modes, this term dominates over the product, $\zeta_{1} \zeta_{2}$, by virtue of Eq. (47), and we get the solution using the scaling of Eq. (46),

$$
\left|A_{1}\right|^{6}=\frac{2}{\left(1-\Gamma_{1} / \Gamma_{2}\right)^{2}}\left(\frac{\Gamma_{1}}{\alpha_{1}}\right)^{2} \frac{\left|B_{1}\right|^{2}}{\Gamma_{1}} .
$$

Using this result, we are able to evaluate the magnitudes of the gains, Eqs. (42)-(44), at the threshold, $\epsilon^{2}=\Gamma_{1} \Gamma_{2}$,

$$
G_{11} \approx G_{12}=\left|\frac{2 \Gamma_{1} \Gamma_{2}}{\text { Det }}\right|^{2}=2\left|A_{1}\right|^{2} \frac{\Gamma_{1}}{\left|B_{1}\right|^{2}} .
$$

Combining equations (47) and (51), we identify the conditions for the nonlinear regime to occur in terms of input power and pump strength,

$$
1-\frac{\epsilon^{2}}{\Gamma_{1} \Gamma_{2}} \ll\left(\frac{\alpha_{1}}{\Gamma_{1}} \frac{\left|B_{1}\right|^{2}}{\Gamma_{1}}\right)^{1 / 3} \ll 1 .
$$

The window for the nonlinear regime is controlled by the parameter $\Gamma_{1} / \alpha_{1}$. The left inequality defines the nonlinear regime, and it is convenient to rewrite it as a lower bound on the input intensity,

$$
\left(1-\frac{\epsilon^{2}}{\Gamma_{1} \Gamma_{2}}\right)^{3} \frac{\Gamma_{1}}{\alpha_{1}} \ll \frac{\left|B_{1}\right|^{2}}{\Gamma_{1}} .
$$

The nonlinear amplification regime may start rather far from the threshold; for instance, for a single-photon input, $\left|B_{1}\right|^{2} / \Gamma_{1}=1$, and $\Gamma_{1} / \alpha_{1}=10$, it starts at $\epsilon>0.77 \sqrt{\Gamma_{1} \Gamma_{2}}$. The right constraint in Eq. (53) allows sufficient room for 
the theory to be valid well above the single-photon input, $1 \ll\left|B_{1}\right|^{2} / \Gamma_{1} \ll \Gamma_{1} / \alpha_{1}$, when $\Gamma_{1} / \alpha_{1} \gg 1$.

The balanced-mode case is of particular interest in the further discussion in the next sections. In this case, the intracavity fields have equal absolute values, $\left|A_{1}\right|=$ $\left|A_{2}\right|=|A|$, according to Eq. (49), which confirms our numerical observation in Fig. 3. The determinant, Eq. (48), is given by the first term, Det $=\left(3 \alpha|A|^{2}\right)^{2}$, since the second term disappears, and then

$$
\begin{aligned}
A_{1} & =|A| e^{-i \pi / 2+i \theta_{B}}, \quad A_{2}=|A| e^{i \pi-i \theta_{B}}, \\
|A|^{5} & =\frac{\sqrt{2} \Gamma^{2}}{(3 \alpha)^{2}} \frac{\left|B_{1}\right|}{\sqrt{\Gamma}} .
\end{aligned}
$$

The nonlinear gains for the balanced modes are determined by this equation, together with Eq. (52).

\section{Two-mode linear amplification}

The amplification of arbitrary detuned input signals can be analyzed in great detail, both analytically and numerically, in the linear amplification regime. The results of this analysis are also relevant for the evaluation of quantum noise and will be used in Sec. IV.

In the linear regime, the intracavity fields generated by the weak input are assumed to be small, $\alpha_{n}\left|A_{n}\right|^{2} \ll \Gamma_{n}$, $\sqrt{\Gamma_{1} \Gamma_{2}-\epsilon^{2}}$, and the Kerr effect is neglected, $\zeta_{n} \approx \delta$. Then the linearized Bogoliubov transformation of Eqs. (41) and (42) can be written in the form

$$
C_{n}(\Delta)=u_{n}(\Delta) B_{n}(\Delta)+v_{n}(\Delta) B_{m}^{\dagger}(-\Delta)
$$

$(m \neq n)$, where the coefficients $u_{1}(\Delta)=\mathcal{V}_{11}(\Delta)$ and $v_{1}(\Delta)=\mathcal{V}_{12}(\Delta)$ for the first mode have the explicit form

$u_{1}(\Delta)=\frac{\left[\delta+\Delta+i\left(\Gamma_{1}-2 \Gamma_{10}\right)\right]\left(\delta-\Delta-i \Gamma_{2}\right)-\epsilon^{2}}{\left(\delta+\Delta+i \Gamma_{1}\right)\left(\delta-\Delta-i \Gamma_{2}\right)-\epsilon^{2}}$,

$v_{1}(\Delta)=\frac{2 i \sqrt{\Gamma_{10} \Gamma_{20}} \epsilon}{\left(\delta+\Delta+i \Gamma_{1}\right)\left(\delta-\Delta-i \Gamma_{2}\right)-\epsilon^{2}}$.

For the second mode, the coefficients are obtained by permutation, $1 \leftrightarrow 2$.

The coefficients satisfy the same relation as in Eq. (43):

$$
\begin{aligned}
\left|u_{n}(\Delta)\right|^{2}-\left|v_{n}(\Delta)\right|^{2} & =1, \\
u_{1}(\Delta) v_{2}(-\Delta)-v_{1}(\Delta) u_{2}(-\Delta) & =0 .
\end{aligned}
$$

These relations not only relate the signal and idler gains, $G_{11}(\Delta)-G_{12}(-\Delta)=1$, but also guarantee preservation of the bosonic commutation relations in the quantum regime [31].

The linear gains diverge at the parametric-instability threshold, Eqs. (27) and (28), as is to be expected.
The linear gain spectra for the signal, $G_{11}(\Delta)=\left|u_{1}(\Delta)\right|^{2}$, and the idler, $G_{12}(-\Delta)=\left|v_{2}(-\Delta)\right|^{2}$, determined by Eq. (57) are shown in Fig. 5. To facilitate a comparison with the experiment, we plot the spectra as functions of the input-signal detuning $\delta_{1}$ from the cavity mode, $\omega_{s}=\omega_{1}+\delta_{1}$, rather than the detuning $\Delta$, which is more convenient for analytics. Their relation is $\delta_{1}=\delta+\Delta$. The idler gain is also plotted vs the input-signal detuning $\delta_{1}$, although the idler is detected at the frequency $\omega_{i}=$ $\omega_{2}+\delta-\Delta=\omega_{2}+2 \delta-\delta_{1}$. Here, we keep finite internal losses, $\Gamma_{n} \neq \Gamma_{n 0}$.

For a pump far detuned from the resonance, $|\delta|>\sqrt{\Gamma_{1} \Gamma_{2}}$, the signal gain in Fig. 5(a) shows a dip centered at $\delta_{1}=0$ due to the presence of internal losses (the green curve in the lower panel); the dip disappears when the signal is detuned away from the resonance, $\delta_{1}>\sqrt{\Gamma_{1} \Gamma_{2}}$, and the scattering is elastic, $G_{11}=1$.

At zero pump detuning, $\delta=0$, the parametric amplification dominates over internal losses, giving rise to a strong gain peak, $G_{11}\left(\delta_{1} \approx 0\right) \gg 1$ (the blue curve). In the
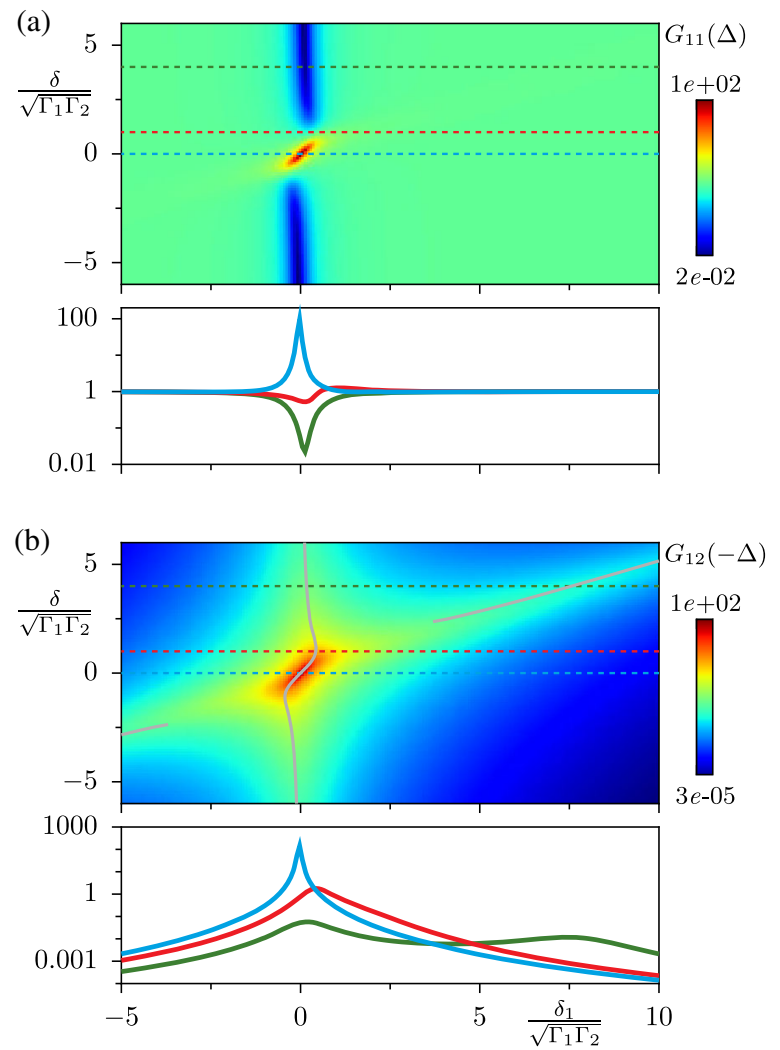

FIG. 5. Linear gain spectra for signal and idler for a detuned input below threshold. (a) Signal gain $G_{11}(\Delta)$ and (b) idler gain $G_{12}(-\Delta)$ as functions of the pump detuning, $\delta$, and the signal detuning, $\delta_{1}=\delta+\Delta$; horizontal dashed lines in the color plots indicate cuts presented in the lower panels, while the thin gray lines in (b) indicate positions of the resonant peaks. $\epsilon=$ $0.95 \sqrt{\Gamma_{1} \Gamma_{2}}, \quad \Gamma_{20}=3 \Gamma_{10}, \Gamma_{1}=1.8 \Gamma_{10}, \Gamma_{2}=4 \Gamma_{10}=(4 / 3) \Gamma_{20}$, $\alpha_{2}=3 \alpha_{1}$, and $\alpha_{1}=\sqrt{\Gamma_{10} \Gamma_{20}} / 100$. 
intermediate region of $\delta$, there is a competition between the two effects. Since the amplification resonance occurs along a tilted line in the $\left(\delta_{1}, \delta\right)$ plane, it shifts the internal-loss resonance away from $\delta_{1}=0$, which therefore features an "avoided crossing" around $\left(\delta_{1}, \delta\right)=0$. Within the avoided crossing, both internal-loss resonance and amplification resonance can coexist in the gain $G_{11}\left(\delta_{1}\right)$, at slightly shifted values of $\delta_{1}$ (the red curve).

The parametric-amplification resonance itself is better resolved in the gain spectrum of the idler, $G_{12}$ [Fig. 5(b)]. Since the idler frequency is out of resonance from the input at $\omega_{1}+\delta_{1}$, it is not affected by the internal-loss resonance, and the $G_{12}$ spectrum is therefore characterized by the parametric amplification alone. The parametricamplification resonances are determined by the local minima of the denominator in Eq. (57). For small pump detunings, $|\delta|<\sqrt{\Gamma_{1} \Gamma_{2}}$, a single resonance exists approximately on the tilted line $\delta_{1}=\delta+\delta\left(\Gamma_{1}-\Gamma_{2}\right) /\left(\Gamma_{1}+\Gamma_{2}\right)$ (the blue curve in the lower panel). For larger values of $|\delta|$, this resonance approaches the line $\delta_{1}=0$ again, while, at the same time, the spectrum develops a shoulder into the blue-detuned (red-detuned) region for $\delta>0(\delta<0)$ (the red curve), from which a second resonance peak eventually arises (the green curve). For balanced modes, $\Gamma_{1}=\Gamma_{2}=\Gamma$, the resonances would be identical to those found for the degenerate parametric amplifier [25], namely, a single resonance at $\delta_{1}=\delta(\Delta=0)$ for $|\delta| \leq \sqrt{\epsilon^{2}+\Gamma^{2}}$, and two split resonances, $\delta_{1}=\delta \pm \sqrt{\delta^{2}-\epsilon^{2}-\Gamma^{2}}$, at $\delta>\sqrt{\epsilon^{2}+\Gamma^{2}}$.

\section{E. Four-mode linear amplification}

Now we turn to the linear response in the presence of a strong intracavity field. Such a field can be generated either by an input signal with another frequency or by parametric oscillation. In this case, the amplification picture changes qualitatively. The strong microwave field in the cavity acts as an additional parametric pump that generates additional idlers, and the picture becomes multimodal.

We first discuss a general situation, then derive the Bogoliubov transformation coefficients within the balanced-mode model. The results directly apply to amplification of noise in the threshold region in the presence of a strong tone. The case of developed parametric oscillation is special, and it is analyzed separately.

Suppose the input signal has the form

$$
B_{1}(t)=\left[B_{1}+b_{1}(t)\right] e^{-i \Delta_{s} t}
$$

i.e., in addition to a stronger signal $B_{1}$ with detuning $\Delta_{S}$ from $\omega_{1}+\delta$, a weaker classical signal $b_{1}(t)$ is applied. Amplification of the strong component is described by Eqs. (38)-(42) (with $\Delta_{S}$ replacing $\Delta$ ).

The presence of a weak input component will generate an addition to the intracavity field, $\left[A_{n}+a_{n}(t)\right] e^{\mp i \Delta_{S} t}$, $a_{n} \ll A_{n}$, which is described using the linearized equations

$$
\begin{aligned}
& i \dot{a}_{1}+\Delta_{S} a_{1}+\bar{\zeta}_{1} a_{1}+i \Gamma_{1} a_{1}+\bar{\epsilon} a_{2}^{*}+2 \alpha A_{1} A_{2}^{*} a_{2}+\alpha_{1} A_{1}^{2} a_{1}^{*} \\
& \quad=\sqrt{2 \Gamma_{10}} b_{1}(t), \\
& i \dot{a}_{2}-\Delta_{S} a_{2}+\bar{\zeta}_{2} a_{2}+i \Gamma_{2} a_{2}+\bar{\epsilon} a_{1}^{*}+2 \alpha A_{1}^{*} A_{2} a_{1}+\alpha_{2} A_{2}^{2} a_{2}^{*} \\
& \quad=\sqrt{2 \Gamma_{20}} b_{2}(t) .
\end{aligned}
$$

Here, we introduce the renormalized $\zeta$ coefficients,

$$
\begin{aligned}
& \bar{\zeta}_{1}=\delta+2 \alpha_{1}\left|A_{1}\right|^{2}+2 \alpha\left|A_{2}\right|^{2}, \\
& \bar{\zeta}_{2}=\delta+2 \alpha_{2}\left|A_{2}\right|^{2}+2 \alpha\left|A_{1}\right|^{2},
\end{aligned}
$$

and the renormalized pump strength,

$$
\bar{\epsilon}=\epsilon+2 \alpha A_{1} A_{2} .
$$

A weak input in the second mode, $b_{2}(t)$, is added for completeness.

In these equations, we see an additional feature: the amplitude, $a_{1}$, of the first mode hybridizes not only with the conjugated amplitude, $a_{2}^{*}$, of the second mode—as it is in the empty cavity-but also with the second-mode amplitude, $a_{2}$, as well as with its own conjugate, $a_{1}^{*}$. This additional feature can be understood as the result of the parametric effect generated by the strong intracavity field with frequencies $\omega_{1,2}+\delta$ : owing to the self-Kerr effect, the second harmonics are generated, $2 \omega_{1,2}+2 \delta$, which act as two additional current pumps producing a degenerate parametric resonance within each mode [15]. Furthermore, the cross-Kerr effect also generates two current pumps, with the combination frequencies $\omega_{2}-\omega_{1}$ and $\omega_{2}+\omega_{1}$, the former producing parametric frequency conversion and the latter generating parametric amplification in addition to the generic flux pump.

The spectrum of the cavity response is illustrated in Fig. 6: the weak signal $(S)$ detuned by $\Delta$ from the strong field of the first mode generates a "primary" idler $\left(I_{1}\right)$ detuned by $-\Delta$ from the strong field of the second mode as well as two "secondary" idlers $\left(I_{2}\right.$ and $\left.I_{3}\right)$ detuned by $\mp \Delta$ from the strong field of the respective modes.

In accord with the structure of Eq. (60), we consider a general form of the input field,

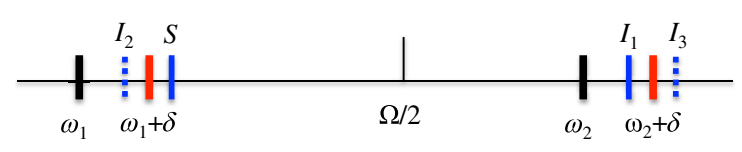

FIG. 6. Four-mode structure of the amplified output field for the detuned signal. Black indicates the cavity resonances, while red marks the parametrically coupled strong-field modes (for $\Delta_{S}=0$ ); the solid blue lines indicate the signal $(S)$ and the primary idler $\left(I_{1}\right)$ with the frequencies $\omega_{1}+\delta+\Delta$ and $\omega_{2}+\delta-\Delta$; the dashed blue lines indicate secondary idlers $\left(I_{2,3}\right)$ with the frequencies $\omega_{1}+\delta-\Delta$ and $\omega_{2}+\delta+\Delta$. 


$$
b_{n}(t)=b_{n}(\Delta) e^{-i \Delta t}+b_{n}(-\Delta) e^{i \Delta t}
$$

and a corresponding form of the intracavity field,

$$
a_{n}(t)=a_{n}(\Delta) e^{-i \Delta t}+a_{n}(-\Delta) e^{i \Delta t} .
$$

The output field, $c_{n}(t)$, will have the same structure.

It is convenient to write the resulting equations for static amplitudes in matrix form, introducing the two-component vectors

$$
\begin{gathered}
a(\Delta)=\left(\begin{array}{c}
a_{1}(\Delta) \\
a_{2}(\Delta)
\end{array}\right), \quad b(\Delta)=\left(\begin{array}{l}
b_{1}(\Delta) \\
b_{2}(\Delta)
\end{array}\right), \\
\mathcal{T}(\Delta) a(\Delta)+\mathcal{E} a^{*}(-\Delta)=\operatorname{diag}\left(\sqrt{2 \Gamma_{n 0}}\right) b(\Delta), \\
\mathcal{T}^{*}(-\Delta) a^{*}(-\Delta)+\mathcal{E}^{*} a(\Delta)=\operatorname{diag}\left(\sqrt{2 \Gamma_{n 0}}\right) b^{*}(-\Delta),
\end{gathered}
$$

where

$$
\mathcal{T}(\Delta)=\left(\begin{array}{cc}
\Delta+\Delta_{S}+\bar{\zeta}_{1}+i \Gamma_{1} & 2 \alpha\left|A_{1} A_{2}\right| e^{i \psi} \\
2 \alpha\left|A_{1} A_{2}\right| e^{-i \psi} & \Delta-\Delta_{S}+\bar{\zeta}_{2}+i \Gamma_{2}
\end{array}\right),
$$

and

$$
\mathcal{E}=\left(\begin{array}{cc}
\alpha_{1}\left|A_{1}\right|^{2} e^{2 i \theta_{1}} & \bar{\epsilon} \\
\bar{\epsilon} & \alpha_{2}\left|A_{2}\right|^{2} e^{2 i \theta_{2}}
\end{array}\right)
$$

$\theta_{1,2}$, and $\psi=\theta_{1}-\theta_{2}$, are the phases of the strong intracavity field. The matrix $\mathcal{T}$ here describes the hybridization of the resonant modes $a_{1}$ and $a_{2}$, while the matrix $\mathcal{E}$ provides the amplification-type coupling to the conjugate pair $\left(a_{1}^{*}, a_{2}^{*}\right)$.

Inverting Eq. (66), we find the intracavity fields and formulate, using the input-output relation, Eq. (8), the Bogoliubov transformation with the matrix coefficients,

$$
c(\Delta)=\hat{U}(\Delta) b(\Delta)+\hat{V}(\Delta) b^{*}(-\Delta) .
$$

These coefficients define the gains for the signal, $G_{11}(\Delta)=$ $\left|U_{11}(\Delta)\right|^{2}$, and the primary idler, $G_{12}(-\Delta)=\left|V_{21}(-\Delta)\right|^{2}$, as well as the gains for the secondary idlers,

$$
\begin{aligned}
G_{11}(-\Delta) & =\left|c_{1}(-\Delta) / b_{1}(\Delta)\right|^{2}=\left|V_{11}(-\Delta)\right|^{2}, \\
G_{12}(\Delta) & =\left|c_{2}(\Delta) / b_{1}(\Delta)\right|^{2}=\left|U_{21}(\Delta)\right|^{2} .
\end{aligned}
$$

\section{Balanced-mode model}

To further analyze the multimode amplification process and to explicitly evaluate the Bogoliubov coefficients, we apply the methods of the multimode-squeezing theory $[39,40]$. This theory operates with a set of "supermodes" that diagonalize the matrix Bogoliubov transformation, Eq. (69).
To do this, one needs to diagonalize the matrices $\mathcal{T}$ and $\mathcal{E}$ in Eq. (66), and this can be relatively simply done for the balanced modes. In this case, the intracavity-field amplitudes are approximately equal for a strong on-resonance input ( $\left.\Delta_{S}=0\right)$, close to the threshold, Eq. (49); $\left|A_{n}\right|=|A|$, hence, $\bar{\zeta}_{n}=\bar{\zeta}$. In the parametric-oscillation regime, the intracavity-field amplitudes are also equal, Eq. (31), and the frequency shift is absent, $\Delta_{0}=0$.

With these approximations, the matrix $\mathcal{T}(\Delta)$ is diagonalized by a unitary rotation,

$$
\mathfrak{U}=\frac{1}{\sqrt{2}}\left(\begin{array}{cc}
e^{i \psi / 2} & e^{i \psi / 2} \\
e^{-i \psi / 2} & -e^{-i \psi / 2}
\end{array}\right),
$$

$$
\mathfrak{U}^{\dagger} \mathcal{T}(\Delta) \mathfrak{U}=\operatorname{diag}\left(\Delta+\zeta_{+}+i \Gamma, \Delta+\zeta_{-}+i \Gamma\right),
$$$$
\zeta_{ \pm}=\bar{\zeta} \pm 2 \alpha|A|^{2}=\delta+(4 \alpha \pm 2 \alpha)|A|^{2} .
$$

Matrix $\mathcal{T}^{*}(-\Delta)$ is diagonalized with the same rotation. Moreover, matrix $\mathcal{E}$ is also diagonalized with the same rotation,

$$
\begin{aligned}
\mathfrak{U}^{\dagger} \mathcal{E} \mathfrak{U}^{*} & =\operatorname{diag}\left(\epsilon_{+}, \epsilon_{-}\right), \\
\epsilon_{ \pm} & = \pm \bar{\epsilon}+\alpha|A|^{2} e^{i \Theta} .
\end{aligned}
$$

In the supermode basis, the field amplitudes take the form $a_{\sigma}(\Delta)=\mathfrak{U}_{\sigma n}^{\dagger} a_{n}(\Delta), \sigma= \pm$, and similarly for the input and output fields, $b_{\sigma}$ and $c_{\sigma}$.

As a result, Eq. (66) splits in the supermode basis into two independent blocks,

$$
\begin{aligned}
\left(\zeta_{\sigma}+\Delta+i \Gamma\right) a_{\sigma}(\Delta)+\epsilon_{\sigma} a_{\sigma}^{*}(-\Delta) & =\sqrt{2 \Gamma} b_{\sigma}(\Delta), \\
\left(\zeta_{\sigma}-\Delta-i \Gamma\right) a_{\sigma}^{*}(-\Delta)+\epsilon_{\sigma}^{*} a_{\sigma}(\Delta) & =\sqrt{2 \Gamma} b_{\sigma}^{*}(-\Delta) .
\end{aligned}
$$

Here, $\Gamma_{n}=\Gamma_{n 0}$ is assumed, neglecting internal losses.

Equation (74) is similar to the linearized Eq. (38) for amplification below threshold, which allows us to readily get the diagonal, two-mode Bogoliubov transformation,

$$
c_{\sigma}(\Delta)=u_{\sigma}(\Delta) b_{\sigma}(\Delta)+v_{\sigma}(\Delta) b_{\sigma}^{*}(-\Delta),
$$

with the Bogoliubov coefficients

$$
\begin{aligned}
& u_{\sigma}(\Delta)=\frac{\left(\zeta_{\sigma}+\Delta-i \Gamma\right)\left(\zeta_{\sigma}-\Delta-i \Gamma\right)-\left|\epsilon_{\sigma}\right|^{2}}{\left(\zeta_{\sigma}+\Delta+i \Gamma\right)\left(\zeta_{\sigma}-\Delta-i \Gamma\right)-\left|\epsilon_{\sigma}\right|^{2}}, \\
& v_{\sigma}(\Delta)=\frac{2 i \Gamma \epsilon_{\sigma}}{\left(\zeta_{\sigma}+\Delta+i \Gamma\right)\left(\zeta_{\sigma}-\Delta-i \Gamma\right)-\left|\epsilon_{\sigma}\right|^{2}} .
\end{aligned}
$$

These coefficients satisfy relations similar to Eq. (58),

$$
\begin{aligned}
\left|u_{\sigma}(\Delta)\right|^{2}-\left|v_{\sigma}(\Delta)\right|^{2} & =1, \\
u_{\sigma}(\Delta) v_{\sigma}(-\Delta)-v_{\sigma}(\Delta) u_{\sigma}(-\Delta) & =0 .
\end{aligned}
$$

Rotating back to the initial basis, we get the matrix Bogoliubov transformation for the fields of the original modes, Eq. (69), where $\hat{U}=\mathfrak{U} \operatorname{diag}\left(u_{+}, u_{-}\right) \mathfrak{U}^{\dagger}$, 


$$
\hat{U}(\Delta)=\frac{1}{2}\left(\begin{array}{ll}
\left(u_{+}+u_{-}\right) & \left(u_{+}-u_{-}\right) e^{i \psi r} \\
\left(u_{+}-u_{-}\right) e^{-i \psi r} & \left(u_{+}+u_{-}\right)
\end{array}\right),
$$

and $\hat{V}=\mathfrak{U} \operatorname{diag}\left(v_{+}, v_{-}\right) \mathfrak{U}^{T}$,

$$
\hat{V}(\Delta)=\frac{1}{2}\left(\begin{array}{ll}
\left(v_{+}+v_{-}\right) e^{i \psi} & \left(v_{+}-v_{-}\right) \\
\left(v_{+}-v_{-}\right) & \left(v_{+}+v_{-}\right) e^{-i \psi}
\end{array}\right) .
$$

These equations define amplification gains for all four modes involved. For the signal and the primary idler, we have the gains

$$
\begin{gathered}
G_{11}(\Delta)=\left|U_{11}(\Delta)\right|^{2}=\frac{1}{4}\left|u_{+}(\Delta)+u_{-}(\Delta)\right|^{2}, \\
G_{12}(-\Delta)=\left|V_{21}(-\Delta)\right|^{2}=\frac{1}{4}\left|v_{+}(-\Delta)-v_{-}(-\Delta)\right|^{2},
\end{gathered}
$$

and, for the secondary idlers,

$$
\begin{gathered}
G_{11}(-\Delta)=\left|V_{11}(-\Delta)\right|^{2}=\frac{1}{4}\left|v_{+}(-\Delta)+v_{-}(-\Delta)\right|^{2}, \\
G_{12}(\Delta)=\left|U_{21}(\Delta)\right|^{2}=\frac{1}{4}\left|u_{+}(\Delta)-u_{-}(\Delta)\right|^{2} .
\end{gathered}
$$

Analyzing these equations with the help of Eq. (76), one can find that the secondary gains are directly proportional to the intracavity-field intensity, $|A|^{2}$, while the gains (80) persist in the limit $|A|^{2}=0$.

Applying the obtained formulas to the case of strong input close to the parametric threshold, Eqs. (48) and (55), we find the Bogoliubov coefficients of the supermodes (for $\delta=0)$,

$$
\begin{aligned}
& u_{\sigma}(\Delta) \approx \frac{-2 \Gamma^{2}+\varsigma_{\sigma}^{2}-\Delta^{2}}{\varsigma_{\sigma}^{2}-\Delta^{2}-2 i \Gamma \Delta}, \\
& v_{\sigma}(\Delta) \approx \frac{2 i \sigma \Gamma^{2}}{\varsigma_{\sigma}^{2}-\Delta^{2}-2 i \Gamma \Delta},
\end{aligned}
$$

with $\varsigma_{+}=\sqrt{3} \zeta$ and $\varsigma_{-}=\zeta / \sqrt{3}, \quad \zeta=3 \alpha|A|^{2}$. These equations show that amplification of the supermode $\sigma=-$ is more efficient, as illustrated in Fig. 7(b).

The linear gain spectra for a detuned input tone $b_{1}(\Delta)$ in the presence of a dominant on-resonance input $B_{1}$ are illustrated in Fig. 7(c). The amplitudes $\left|A_{1,2}\right|^{2}$ of the cavity field generated by $B_{1}$ are shown in Fig. 7(a) and are used in Eqs. (66)-(69) to compute the linear gains. Around the threshold, $\epsilon \approx \Gamma$, the cavity amplitudes are approximately equal, $\left|A_{1}\right|^{2} \approx\left|A_{2}\right|^{2}$, and fulfill the conditions of Eq. (47).

\section{F. Four-mode linear amplification in the oscillator regime}

Quite a different situation occurs in the parametricoscillator regime above the threshold. Given equations (a)

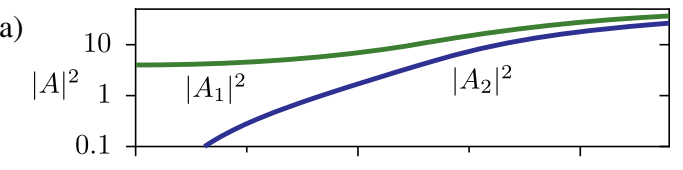

(b)
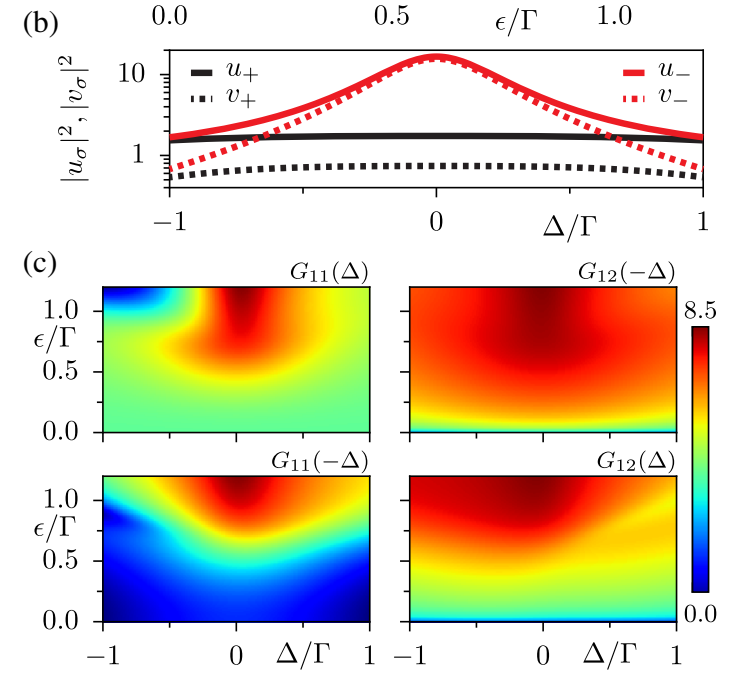

FIG. 7. Linear gain spectra for balanced modes obtained from Eqs. (66)-(69) in the presence of the strong on-resonance input tone $\left|B_{1}\right|^{2}=2 \Gamma, \theta_{B}=0$. (a) Cavity-field amplitudes $\left|A_{1,2}\right|^{2}$ generated by $B_{1}$ vs the pump strength $\epsilon$. (b) Bogoliubov coefficients $\left|u_{\sigma}\right|^{2},\left|v_{\sigma}\right|^{2}$ of supermodes vs detuning $\Delta$ for $\epsilon=0.95 \Gamma$, computed from Eq. (76) using the approximation $|A|^{2}=\left(\left|A_{1}\right|^{2}+\left|A_{2}\right|^{2}\right) / 2$. (c) Linear gains $G_{11}(\Delta), G_{12}(-\Delta)$, $G_{11}(-\Delta), G_{12}(\Delta)$ vs the input signal detuning $\Delta$ and the pump strength $\epsilon$, for the additional weak signal $b_{1}(\Delta)$ detuned by $\Delta$ from $\omega_{1} . \delta=0, \Gamma_{2}=\Gamma_{1}, \alpha_{2}=\alpha_{1}=\sqrt{\Gamma_{1} \Gamma_{2}} / 100$, and $\Gamma_{n}=\Gamma_{n 0}$.

for the oscillation intensity and phase, Eqs. (31)-(34), we compute the determinant of Eq. (74) for the supermodes,

$\operatorname{Det}_{+}(\Delta)=4\left(\epsilon^{2}-\delta \sqrt{\epsilon^{2}-\Gamma^{2}}-\Gamma^{2}\right)-\Delta(\Delta+2 i \Gamma)$,

$\operatorname{Det}_{-}(\Delta)=-\Delta(\Delta+2 i \Gamma)$.

Here, we see that, while $\operatorname{Det}_{+}(\Delta)$ is finite everywhere except at the threshold point, $\operatorname{Det}_{-}(\Delta=0)$ turns to zero for all pump strengths above the threshold. Consequently, the Bogoliubov coefficients of the supermode $\sigma=-$ are singular at the oscillation frequency $\Delta=0$. Furthermore, they grow with increasing pump strength,

$$
u_{-}(\Delta) \approx v_{-}(\Delta) \approx \frac{(4 / 3) i \Gamma \epsilon}{\Delta(\Delta+2 i \Gamma)}, \quad \epsilon \gg \Gamma .
$$

At the same time, the regular coefficients for the supermode $\sigma=+$ behave qualitatively similarly to the degenerateresonance case [25],

$$
u_{+} \approx 1, \quad v_{+} \approx-\frac{\Gamma^{2}}{2 \epsilon^{2}}, \quad \epsilon \gg \Gamma .
$$



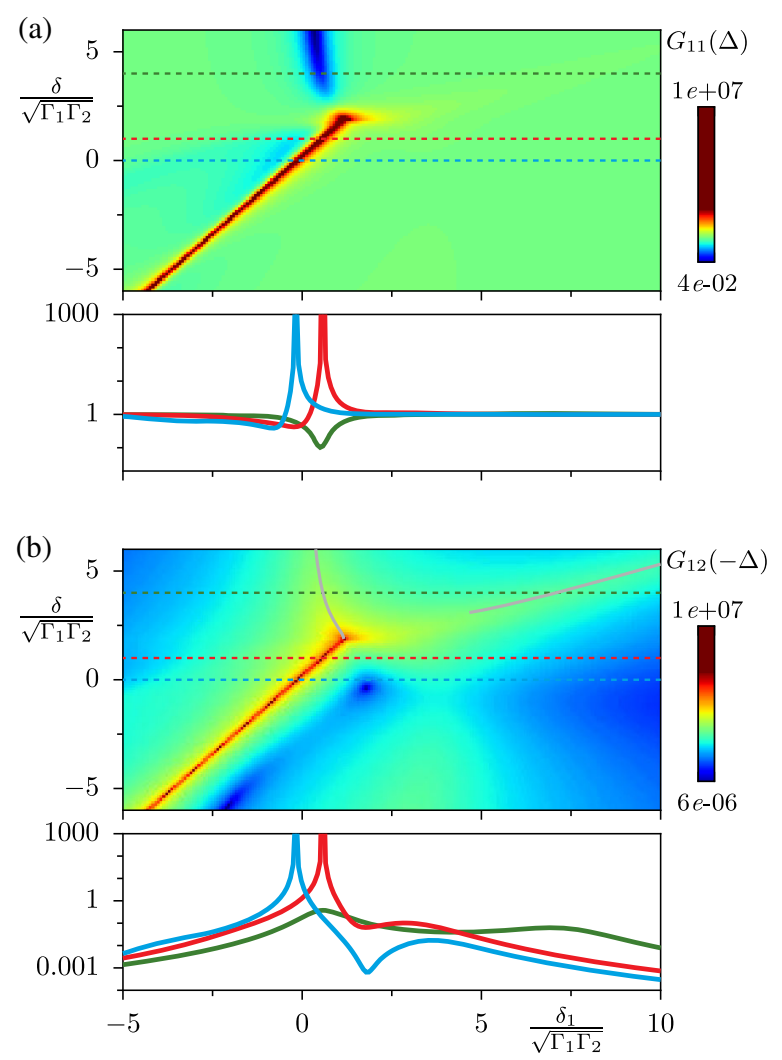

FIG. 8. Linear gain spectra for the signal and the primary idler above threshold, obtained from Eqs. (66)-(69). (a) The signal gain $G_{11}(\Delta)$ and (b) the primary idler gain $G_{12}(-\Delta)$ vs the inputsignal detuning $\delta_{1}$ and the pump detuning $\delta$; the horizontal dashed lines in the color plots indicate cuts presented in the lower panels, while the thin gray lines in (b) indicate the positions of resonant peaks. The bright region indicates the resonant peak at the radiation frequency $\Delta_{0}(\delta)$. The blue strip outside parametricoscillation region, $\delta>\delta_{\text {th }} \approx 2 \sqrt{\Gamma_{1} \Gamma_{2}}$, in (a) is due to internal losses, while the blue strip within the parametric-oscillation region in (b) is due to energy transfer to the secondary idlers; see Fig. 9. $\epsilon=2 \sqrt{\Gamma_{1} \Gamma_{2}}, \Gamma_{20}=3 \Gamma_{10}, \Gamma_{1}=1.8 \Gamma_{10}, \Gamma_{2}=4 \Gamma_{10}=$ $(4 / 3) \Gamma_{20}, \alpha_{2}=3 \alpha_{1}$, and $\alpha_{1}=\sqrt{\Gamma_{10} \Gamma_{20}} / 100$.

The anomalous behavior of the supermode $\sigma=-$ implies that it solely defines all of the gains close to the oscillation frequency, and well above the threshold at all frequencies. In the latter region, all four gains have approximately the same asymptotic scaling,

$G_{11}( \pm \Delta) \approx G_{12}( \pm \Delta) \approx \frac{(4 / 9) \Gamma^{2} \epsilon^{2}}{\Delta^{2}\left(\Delta^{2}+4 \Gamma^{2}\right)}, \quad \epsilon \gg \Gamma$.

In Figs. 8 and 9, the linear gain spectra above the threshold are shown for different pump- and input-signal detunings. The plots are made using the general equations (66)-(69) to illustrate the behavior of unbalanced modes in the presence of internal damping. We remind the reader that $\Delta$ in the parametric-oscillation regime, $\delta<\delta_{\mathrm{th}}$, refers to the deviation from the frequency of the oscillation,
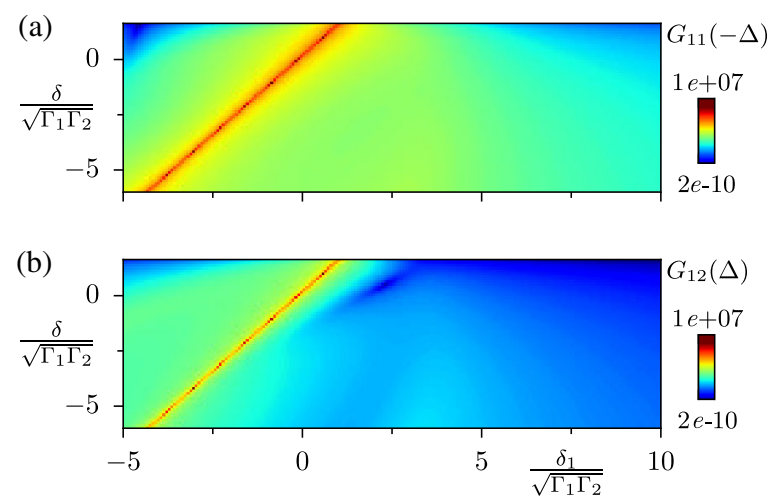

FIG. 9. Linear gain spectra for the secondary idlers above the threshold, (a) $G_{11}(-\Delta)$ and (b) $G_{12}(\Delta)$; these idlers appear at $\delta<\delta_{\text {th }} \approx 2 \sqrt{\Gamma_{1} \Gamma_{2}}$, in addition to the signal and the primary idler presented in Fig. 8. The parameters are the same as those used in Fig. 8.

$\Delta_{0}$ [Eq. (30)]. For the signal detuning, we again use the notation $\delta_{1}=\omega_{s}-\omega_{1}$ rather than $\Delta$.

Figure 8 illustrates the linear gain spectra of the signal, $G_{11}(\Delta)$, and the primary idler, $G_{12}(-\Delta)$. Outside the parametric-oscillation region, $\delta>\delta_{\mathrm{th}}=1.9 \sqrt{\Gamma_{1} \Gamma_{2}}$, the cavity is empty and the detuning $\Delta$ here refers to the reference frequency $\omega_{1}+\delta$, corresponding to the input detuning $\delta_{1}=\delta+\Delta$. The primary idler has the frequency $\omega_{2}+2 \delta-\delta_{1}$.

The spectrum is similar to that discussed and shown in Fig. 5, where $\delta_{\text {th }}$ now plays the role of $\delta=0$ in the latter. For $\delta>\delta_{\text {th }}$, the spectrum $G_{12}(-\Delta)$ has two resonance peaks [the green curve in the lower panel of Fig. 5(b)]. One of these peaks is accompanied by a dip in $G_{11}(\Delta)$ stemming from the internal losses. When approaching $\delta_{\text {th }}$, this dip gradually becomes shallower due to the proximity of the parametric-oscillation threshold, while one of the resonance peaks of $G_{12}(-\Delta)$ disappears.

For balanced modes where $\Delta_{0}=0$, these resonance positions are similar to the degenerate resonance [25]: a single resonance at $\delta_{1}=\delta$, i.e., $\Delta=0$, for $\delta_{\text {th }} \leq \delta \leq \sqrt{\epsilon^{2}+\Gamma^{2}}$, which splits into $\delta_{1}=\delta \pm \sqrt{\delta^{2}-\epsilon^{2}-\Gamma^{2}}$ at $\delta=\sqrt{\epsilon^{2}+\Gamma^{2}}$.

At $\delta=\delta_{\text {th }}$, the parametric resonance lies at $\delta_{1}=$ $\delta_{\text {th }}\left[1+\left(\Gamma_{1}-\Gamma_{2}\right) /\left(\Gamma_{1}+\Gamma_{2}\right)\right]$, which coincides there with the parametric-oscillation detuning at the threshold, $\delta_{1}=\delta_{\text {th }}+\Delta_{0}\left(\delta_{\text {th }}\right)$. Owing to the proximity to the parametric-oscillation state, the resonance is very strong.

Within the parametric-oscillation region $\delta<\delta_{\text {th }}$, the reference frequencies shift to $\omega_{1}+\delta+\Delta_{0}$ and $\omega_{2}+\delta-\Delta_{0}$, respectively. Therefore, the relation of the input detuning $\delta_{1}$ to $\Delta$ becomes $\delta_{1}=\delta+\Delta_{0}+\Delta$, and the idler frequency is $\omega_{2}+2 \delta-\delta_{1}$. The gains of the signal and the idler show strong resonance where the input frequency coincides with the frequency of the parametric oscillation, $\delta_{1}=\delta+\Delta_{0}(\delta)$, in the whole parametric-oscillation region. This observation agrees with the analytical result derived for the balanced modes, Eq. (86). Such behavior is drastically different from 


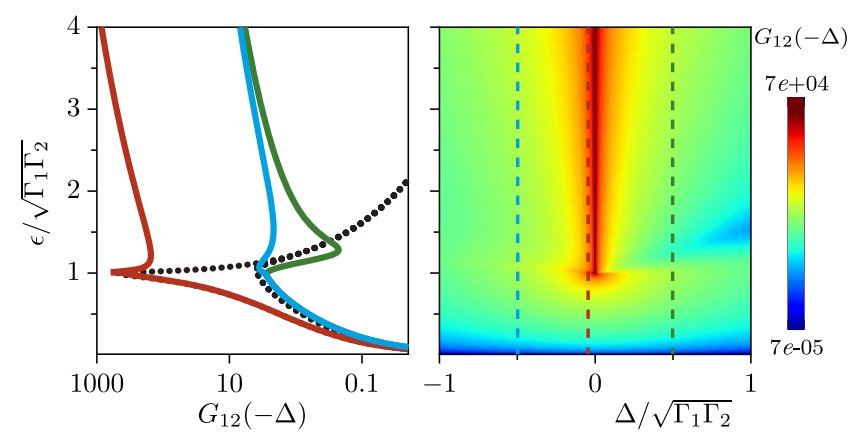

FIG. 10. Linear idler gain $G_{12}(-\Delta)$ vs the input signal detuning $\Delta$ and the pump strength $\epsilon$, obtained from Eqs. (66)-(69). Gain divergence above threshold occurs when the input is in resonance with the parametric oscillation, $\Delta=0$. The left panel shows cuts indicated by dashed lines on the right panel. $\delta=0, \Gamma_{2}=3 \Gamma_{1}$, $\alpha_{2}=3 \alpha_{1}=3 \sqrt{\Gamma_{1} \Gamma_{2}} / 100, \Gamma_{n}=\Gamma_{n 0}$. For a comparison, the idler gain $G_{12}(-\Delta)$ for a degenerate amplifier is presented as dotted lines (with the parameters $\alpha=3 \sqrt{\alpha_{1} \alpha_{2}}, \Gamma=\sqrt{\Gamma_{1} \Gamma_{2}}$ ).

the degenerate case, where the linear gain diverges only at the threshold.

The behavior of the secondary idler gains, $G_{11}(-\Delta)$ and $G_{12}(\Delta)$, are illustrated in Fig. 9. These idlers are measured at the frequencies $\omega_{1}+2 \delta-\delta_{1}+2 \Delta_{0}$ and $\omega_{2}+\delta_{1}-2 \Delta_{0}$, respectively. Because of the distribution of the input power over the four output modes, the spectral characteristics for each of the idlers become more complicated than for the conventional two-mode amplification below threshold, such as nonmonotonic behavior away from the resonance; this is e.g. also seen in $G_{12}(-\Delta)$ at $\delta=0$ (the blue curve) in Fig. 8.

Figure 10 illustrates the linear gain spectrum of the primary idler, $G_{12}(-\Delta)$, as a function of the pump strength $\epsilon$ and the input detuning. It is calculated from Eqs. (66)-(69), where the parametric-oscillation amplitudes $A_{10}$ and $A_{20}$ are taken into account above threshold, $\epsilon>\sqrt{\Gamma_{1} \Gamma_{2}}$, while, below threshold, with $A_{n}=0$, the linear gains are identical to those in Eqs. (56) and (57). Above threshold, the gain diverges when the input is in resonance with the parametric oscillation for all pump strengths, $\delta_{1}=\delta+\Delta_{0}(\epsilon)$, in accord with Figs. 8 and 9. Another interesting feature is that the gain does not decrease to zero with increasing pump strength, as it does in the degenerate case. In fact, all of the gains increase with $\epsilon$, in agreement with Eq. (86).

\section{Phase locking and regularization of gain divergence}

The divergence of the linear response in the oscillation regime found in Eqs. (83) and (84) is a hallmark of nondegenerate parametric resonance [29,34-36]. The divergence is related to the degeneracy of the free oscillation state with respect to the mode-relative phase. Indeed, the divergence is caused by the zero value of the determinant of the supermode dynamical matrix, which implies that this matrix has at least one zero eigenvalue. On the other hand, the zero eigenvalue of the linearized dynamical matrix indicates an indifferent equilibrium of the oscillator state.

To eliminate the divergence, one needs to go beyond the linear approximation, which can be done for an onresonance input at $\Delta=0$ (we remind the reader that the nonlinear response at finite detuning is nonstationary).

Our strategy will be to first transform the original nonlinear equations, Eq. (38), to the supermode basis, and then to linearize the equations with respect to $a_{+}$while keeping the nonlinear terms in $a_{-}$.

In terms of the supermode amplitudes for the balanced modes, Eq. (38) takes the form

$$
\left(\frac{3 \alpha}{2}\left|A_{\sigma}\right|^{2}+\alpha\left|A_{-\sigma}\right|^{2}+i \Gamma\right) A_{\sigma}+\left(\sigma \epsilon-\frac{\alpha}{2} A_{-\sigma}^{2}\right) A_{\sigma}^{*}=\sqrt{2 \Gamma} b_{\sigma},
$$

where we introduce the notation $b_{\sigma}=\mathfrak{U}_{\sigma n}^{\dagger} B_{n}$ and assume, for simplicity, that $\delta=0$ and $\Gamma=\Gamma_{0}$.

The free solution is nontrivial only for the supermode $\sigma=+$,

$$
A_{+0}=\sqrt{2}\left|A_{0}\right| e^{i \Theta_{0} / 2}, \quad A_{-0}=0 .
$$

By separating the free solution, $A_{\sigma}=A_{\sigma 0}+a_{\sigma}$, and linearizing the equation for $\sigma=+$, we reproduce the first equation in Eq. (74). Linearization of the equation for $\sigma=-$ with respect to $a_{+}$yields a nonlinear extension of the second equation in Eq. (74),

$$
\left(\frac{3 \alpha}{2}\left|a_{-}\right|^{2}+\zeta_{-}+i \Gamma\right) a_{-}+\epsilon_{-} a_{-}^{*}=\sqrt{2 \Gamma} b_{-} .
$$

It is convenient to introduce, for brevity, the quantity

$$
Q=\zeta_{-}+i \Gamma=\frac{2}{3} \sqrt{\epsilon^{2}-\Gamma^{2}}+i \Gamma
$$

and variables $\bar{a}_{-}=a_{-} e^{-i \Theta_{0} / 2}$ and $\bar{b}_{-}=b_{-} e^{-i \Theta_{0} / 2}$. The equation then takes the form

$$
\frac{3 \alpha}{2}\left|\bar{a}_{-}\right|^{2} \bar{a}_{-}+Q \bar{a}_{-}+Q \bar{a}_{-}^{*}=\sqrt{2 \Gamma} \bar{b}_{-} .
$$

Following the standard way of inverting this equation and substituting the solution into the input-output relation, we obtain the nonlinear Bogoliubov transformation

$$
\begin{aligned}
\bar{c}_{-} & =\frac{\left(\frac{3 \alpha}{2}\left|\bar{a}_{-}\right|^{2}+Q^{*}\right)^{2}-|Q|^{2}}{\operatorname{Det}} \bar{b}_{-}+\frac{2 i \Gamma Q}{\operatorname{Det}} \bar{b}_{-}^{*} \\
& =\bar{u}_{-} \bar{b}_{-}+\bar{v}_{-} \bar{b}_{-}^{*}, \\
\operatorname{Det} & =\frac{3 \alpha}{2}\left|a_{-}\right|^{2}\left(\frac{3 \alpha}{2}\left|a_{-}\right|^{2}+Q+Q^{*}\right) .
\end{aligned}
$$


One can straightforwardly verify that the relation between the nonlinear Bogoliubov coefficients $\left|\bar{u}_{-}\right|^{2}-\left|\bar{v}_{-}\right|^{2}=1$ still holds. Summarizing, more accurate calculation including nonlinearity results in a regular response to the onresonance input.

This result, however, creates a problem. The "input" amplitude, $\bar{b}_{-}=\left(e^{-i \psi / 2} b_{1}-e^{i \psi / 2} b_{2}\right) e^{-i \Theta_{0} / 2} / \sqrt{2}$, in Eq. (92) contains the uncertain phase $\psi$, which is also present in the Bogoliubov coefficients in the original modes, Eq. (78). This phase has been treated thus far as a free parameter, but, in fact, it is locked in the driven oscillator, as is mentioned in Sec. III C 1.

To reveal the locking effect, we make a polar decomposition of the total intracavity fields, $A_{1,2}=r_{1,2} e^{i(\Theta \pm \psi) / 2}$, and the supermode amplitudes then read

$$
A_{ \pm}=\frac{r_{1} \pm r_{2}}{\sqrt{2}} e^{i \Theta / 2}
$$

Using this representation, we are able to formulate the constraint, $\operatorname{Im}\left(A_{-} / A_{+}\right)=0$, which reads, for $\bar{a}_{-}$,

$$
\operatorname{Im} \frac{\bar{a}_{-}}{\sqrt{2}\left|A_{0}\right|+\bar{a}_{+}}=0,
$$

with $\bar{a}_{+}=a_{+} e^{-i \Theta_{0} / 2}$, or

$$
\sqrt{2}\left|A_{0}\right| \operatorname{Im} \bar{a}_{-}+\operatorname{Im}\left(\bar{a}_{-} \bar{a}_{+}^{*}\right)=0 .
$$

This nonlinear equation establishes a missing relation between the oscillation relative phase and the on-resonance input amplitudes.

Having in hand Eq. (96), we are now able to construct a consistent solution to Eq. (91) in the linear approximation. In this limit, Eq. (96) reduces to $\operatorname{Im} \bar{a}_{-}=0$, which yields the linearized solution

$$
\bar{a}_{-}=\operatorname{Re} \bar{a}_{-}=\sqrt{\frac{\Gamma}{2}} \frac{\bar{b}_{-}}{Q},
$$

and the constraint on the source, $\operatorname{Im}\left(Q^{*} \bar{b}_{-}\right)=0$, or

$$
Q^{*} b_{-}=Q e^{i \Theta_{0}} b_{-}^{*} .
$$

One can explicitly extract the phase factor from this equation and express it through the mode inputs, e.g., for the single-mode input, $B_{2}=0$,

$$
e^{i \psi}=e^{-i \Theta_{0}} \frac{Q^{*}}{Q} \frac{B_{1}}{B_{1}^{*}} .
$$

Thus, the uncertain relative phase of the free oscillator is locked by the on-resonant input and defined by the phase of this input.
Owing to the phase-locking effect, the on-resonance output field becomes regular,

$$
c_{-}=\frac{Q+Q^{*}}{2 Q} b_{-},
$$

in the linear approximation.

In fact, the phase locking can also be used to eliminate the divergence of the linear response to a detuned input at $\Delta \rightarrow 0$. To this end, we note that the singular gains in Eq. (86) are defined for a single harmonic input, $b_{-}(\Delta)$, at detuning $\Delta$, while $b_{-}^{*}(-\Delta)$ at detuning $-\Delta$ is assumed to be zero [cf. gain definitions at finite detuning, e.g., Eqs. (80) and (81)]. However, at zero detuning, a regular Bogoliubov transformation results from the interference of the input amplitudes, $b_{-}(0)$ and $b_{-}^{*}(0)$.

Taking this remark into account, we consider a slightly broadened input whose spectrum contains contributions of both positive and negative harmonics in $\Delta$. Expressing the Bogoliubov transformation in Eqs. (75) and (76) through $Q$,

$$
c_{-}(\Delta)=\frac{2 i \Gamma\left[Q^{*} b_{-}(\Delta)-Q e^{i \Theta_{0}} b_{-}^{*}(-\Delta)\right]+\Delta^{2} b_{-}(\Delta)}{\Delta(\Delta+2 i \Gamma)},
$$

we insert Eq. (98) into the square brackets to get

$$
Q^{*}\left[b_{-}(\Delta)-b_{-}(0)\right]-Q e^{i \Theta_{0}}\left[b_{-}^{*}(-\Delta)-b_{-}^{*}(0)\right] .
$$

Assuming a spectrally smooth input, $b_{-}(\Delta)-b_{-}(0) \approx$ $b_{-}^{\prime}(0) \Delta$, we arrive at the output field,

$c_{-}(\Delta) \approx \frac{2 i \Gamma\left[Q^{*} b_{-}^{\prime}(0)+Q e^{i \Theta} b_{-}^{\prime *}(0)\right]+\Delta b_{-}(\Delta)}{2 i \Gamma+\Delta}$,

which is regular at $\Delta=0$.

\section{QUANTUM FLUCTUATIONS}

In this section, we consider quantum fluctuations of the output field of the nondegenerate parametric amplifier. We use the results of the classical analysis in Sec. III to compute the noise spectral densities and evaluate the effect of the noise squeezing and the output signal-to-noise ratio.

The amplifier output consists of the sum of the classical signals studied so far, $C_{n}(t)$, and the quantum noise represented by the bosonic operators, $c_{n}(t)$, in both parametrically coupled modes. The output is concentrated around the frequencies of the respective modes, $\omega_{n}+\delta$, within the bandwidths, $\Gamma_{n}$. The measurement of the output microwave signals are performed via homodyne detection [42], when the output fields are mixed with the fields of two local oscillators, $A_{\mathrm{LO}} e^{i\left(\omega_{n}+\delta\right) t+i \theta_{n}}+$ c.c., and low-frequency envelopes are filtered out, yielding the quadratures 


$$
\begin{aligned}
X(t) & =\sum_{n=1,2}\left[X_{n}^{\theta_{n}}(t)+x_{n}^{\theta_{n}}(t)\right] \\
& =\sum_{n}\left\{\left[C_{n}(t)+c_{n}(t)\right] e^{-i \theta_{n}}+\text { H.c. }\right\}
\end{aligned}
$$

(in this section, we use the notation $\theta_{1,2}$ for the localoscillator phases, rather than the phases of the classical oscillator amplitudes, which are not discussed here). The spectral power for the obtained quadratures is defined as

$$
\begin{aligned}
P(\Delta) & =\lim _{T \rightarrow \infty} \frac{1}{2 T}\left|\int_{-T}^{T} d t X(t) e^{i \Delta t}\right|^{2} \\
& =P_{0}(\Delta)+S(\Delta),
\end{aligned}
$$

where $P_{0}(\Delta)$ refers to the coherent-signal component and reads, for a single input tone with frequency $\omega_{1}+\delta$,

$$
P_{0}(\Delta)=2 \pi \delta(\Delta)\left[\sum_{n}\left(C_{n} e^{-i \theta_{n}}+C_{n}^{*} e^{i \theta_{n}}\right)\right]^{2} .
$$

The second term in Eq. (105) refers to the noise component and is commonly quantified with the squeezing spectral density [43],

$$
S(\Delta)=\int_{-\infty}^{\infty} d t e^{i \Delta t}\langle x(t) x(0)\rangle=\sum_{n m} S_{n m}^{\theta_{n} \theta_{m}}(\Delta) .
$$

Noise squeezing is manifested in the anisotropy of the quadrature spectral density with respect to the localoscillator phases, which results from the interference between the signal and the idler. Noise squeezing in a single mode is not possible in the two-mode linear amplification regime. On the other hand, under the fourmode nonlinear amplification, noise squeezing is detectable even in the single-mode measurement.

\section{A. Squeezing spectral density}

The partial components of the squeezing spectral density in Eq. (107) can be expressed through Fourier harmonics of the noise quadratures,

$S_{n m}^{\theta_{n} \theta_{m}}(\Delta)=\int_{-\infty}^{\infty} d \Delta^{\prime}\left\langle x_{n}^{\theta_{n}}(\Delta) x_{m}^{\theta_{m}}\left(\Delta^{\prime}\right)\right\rangle=S_{m n}^{\theta_{m} \theta_{n}}(\Delta)$,

where

$$
\begin{aligned}
x_{n}^{\theta}(\Delta) & =\int_{-\infty}^{\infty} \frac{d t}{\sqrt{2 \pi}} e^{i \Delta t} x_{n}^{\theta}(t) \\
& =c_{n}(\Delta) e^{-i \theta}+c_{n}^{\dagger}(-\Delta) e^{i \theta} .
\end{aligned}
$$

Hereafter, the averaging is assumed to be over the input vacuum state.

To compute the correlation functions in Eq. (108), we use the linearized Bogoliubov transformation either in the form of Eqs. (56) and (57) - for the two-mode squeezingor in the form of Eqs. (69), (78), and (79)—for the fourmode squeezing of balanced modes. In the general case of unbalanced modes, Eqs. (66)-(69) have to be used.

Owing to the linear form of the Bogoliubov transformation, it can be readily extended to the quantum case by replacing the classical amplitudes with the bosonic operators. The Bogoliubov transformation preserves the canonical commutation relations; i.e., the output operators are bosonic provided that the input operators are bosonic [31],

$\left[c_{n}(\Delta), c_{m}^{\dagger}\left(\Delta^{\prime}\right)\right]=\left[b_{n}(\Delta), b_{m}^{\dagger}\left(\Delta^{\prime}\right)\right]=\delta_{n m} \delta\left(\Delta-\Delta^{\prime}\right)$.

This property is fulfilled in the absence of internal losses (otherwise, one should add additional input noise channels), and, in the two-mode case, it follows from the properties of the $u-v$ coefficients in Eq. (58). In the four-mode case, the supermode operators respect the commutation relation due to similar relations between the $u-v$ coefficients in Eq. (77), and, since the supermodes are connected to the original modes via unitary rotation, the commutation relations hold also for the mode operators (cf. Ref. [39]).

The latter argument does not, however, apply to the parametric-oscillator regime. In this case, the Bogoliubov coefficients in Eqs. (78) and (79) depend on the free oscillation phase $\psi$. As we found in the classical analysis, this phase is defined by the input, and therefore it is susceptible to the input noise. Therefore, this phase undergoes quantum fluctuation, which is correlated with the input quantum noise. As a result, the structure of the output noise correlation functions must be qualitatively different from that in the subthreshold region, and from conventional two-mode squeezing for the degenerate oscillator [25]. This topic requires a separate treatment, which goes beyond the scope of this paper.

\section{B. Weak signal, two-mode noise squeezing}

First, we analyze Eqs. (107) and (108) in the case of two-mode squeezing for amplification of a weak signal below threshold. In this case, the input-output relation [Eq. (56)] with linearized Bogoliubov coefficients [Eq. (57)] applies independently to both signal and noise. For equal local-oscillator phases, $\theta_{1}=\theta_{2}=\theta$, we get

$$
\begin{aligned}
S_{n}^{\theta}(\Delta) & =\left|u_{n}(\Delta)\right|^{2}+\left|v_{n}(-\Delta)\right|^{2}, \\
S_{12}^{\theta}(\Delta) & =u_{1}(\Delta) v_{2}(-\Delta) e^{-2 i \theta}+u_{2}^{*}(\Delta) v_{1}^{*}(-\Delta) e^{2 i \theta} . \\
& =\left[S_{21}^{\theta}(\Delta)\right]^{*} .
\end{aligned}
$$

The full spectral density can be transformed, after regrouping using the symmetry properties of the $u-v$ coefficients, to the form 
$S^{\theta}(\Delta)=\sum_{ \pm}\left[e^{-2 r( \pm \Delta)}+2 \sinh 2 r( \pm \Delta) \cos ^{2}\left(\frac{\chi( \pm \Delta)}{2}-\theta\right)\right]$,

where we introduce the squeezing parameter, $r(\Delta)$, through the relation

$$
\left|v_{1}(\Delta)\right|=\left|v_{2}(-\Delta)\right|=\sinh r(\Delta),
$$

and the phase

$$
\chi(\Delta)=\arg \left[u_{1}(\Delta) v_{2}(-\Delta)\right]
$$

As one can see from Eq. (111), the output noise mixed with only one local oscillator, $S_{1}(\Delta)$, does not contain $\theta$-dependent interference terms, implying that the noise of a single mode is not squeezed but merely amplified. This behavior results from the large difference in the signal and idler frequencies. In the limit of strong (but still linear) amplification, $G_{11} \approx G_{12} \gg 1$, close to the threshold, $\sqrt{\Gamma_{1} \Gamma_{2}}-\epsilon \ll \Gamma_{n}(\delta=0)$, the spectral density is proportional to the gain,

$$
S_{1}(\Delta) \approx 2\left|u_{1}(\Delta)\right|^{2}=2 G_{11}(\Delta)
$$

and has a sharp peak localized at small $|\Delta| \lesssim \sqrt{\Gamma_{1} \Gamma_{2}}-\epsilon \ll \Gamma_{n}$, shown in Fig. 11.

In order to achieve noise squeezing, one needs to employ two local oscillators, in which case the full noise, Eq. (112), includes the interference term, and the amplification effect described by this term can be canceled by the appropriate choice of the local-oscillator phase $\theta$,

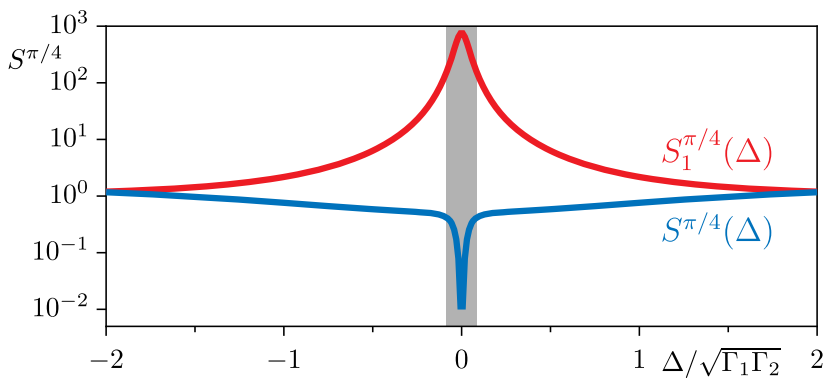

FIG. 11. Linear squeezing spectra $S_{1}^{\pi / 4}(\Delta)$ and $S^{\pi / 4}(\Delta)$ vs input detuning $\Delta$ for two-mode squeezing in an empty cavity. Individual mode spectral density $S_{1}^{\pi / 4}(\Delta)$ from Eq. (111) (the red curve). Full two-mode spectral density $S^{\pi / 4}(\Delta)=$ $\left(S_{1}^{\pi / 4}+S_{2}^{\pi / 4}+S_{12}^{\pi / 4}+S_{21}^{\pi / 4}\right)(\Delta)$ from Eq. (111) (the blue curve). The gray shaded area marks the region $|\Delta|<\sqrt{\Gamma_{1} \Gamma_{2}}-\epsilon$. The local-oscillator phase is chosen in the maximum squeezing direction, $\theta=\pi / 4$ [Eq. (116)], using $\chi(0)=-\pi / 2 . \epsilon=$ $0.95 \sqrt{\Gamma_{1} \Gamma_{2}}, \delta=0, \Gamma_{2}=3 \Gamma_{1}, \alpha_{2}=3 \alpha_{1}=3 \sqrt{\Gamma_{1} \Gamma_{2}} / 100$, and $\Gamma_{n}=\Gamma_{n 0}$.

$$
\theta=\frac{\pi}{2}+\frac{\chi(0)}{2}
$$

In this maximum-squeezing direction, the spectral density has a sharp dip, as illustrated in Fig. 11, with a minimum value, $S_{\min }(0) \approx 1 /\left[2 G_{11}(0)\right]$, while, in the $\pi / 2$-shifted maximum-amplification direction, it has a sharp peak with a maximum value of $S_{\max }(0) \approx 8 G_{11}(0)$.

\section{Signal-to-noise ratio}

The efficiency of amplification of the signal with respect to the noise is quantified with the signal-to-noise ratio,

$$
\mathrm{SNR}=\frac{\max \overline{P_{0}^{\theta}}}{\overline{S^{\theta}}}
$$

where the overbar indicates integration over some bandwidth $(-\bar{\Delta} / 2, \bar{\Delta} / 2)$.

In the case of the homodyne detection with one local oscillator, the signal-output amplitude is $C_{1}=u_{1}(0) B_{1}=$ $-\sqrt{G_{11}(0)} B_{1}$. The resulting spectral power of the signal is, according to Eq. (106),

$$
\overline{P_{0}^{\theta}}=8 \pi G_{11}(0) \cos ^{2}\left(\theta_{B}-\theta\right)\left|B_{1}\right|^{2} .
$$

Integrating the noise over a small bandwidth, $\bar{\Delta} / 2 \ll$ $\sqrt{\Gamma_{1} \Gamma_{2}}-\epsilon$, we get $\overline{S_{1}} \approx 2 G_{11}(0) \bar{\Delta}$ and

$$
\mathrm{SNR}=4 \pi \frac{\left|B_{1}\right|^{2}}{\bar{\Delta}} .
$$

This is half of the input SNR value. The factor $1 / 2$ reflects the noise added by the idler.

Homodyne detection with two local oscillators adds the idler contribution also to the signal output, $C_{2}=v_{2}(0) B_{1}^{*}$, which results in the spectral power,

$\overline{P_{0}^{\theta}}=32 \pi G_{11}(0) \cos ^{2}\left(\theta_{B}+\frac{\pi}{4}\right) \times \cos ^{2}\left(\frac{\chi(0)}{2}-\theta\right)\left|B_{1}\right|^{2}$.

Comparing this result with Eq. (112), we find that the maximum-squeezing direction for the noise and the signal coincide. For the maximum-amplification direction, the signal-to-noise ratio,

$$
\mathrm{SNR}=8 \pi \frac{\left|B_{1}\right|^{2}}{\bar{\Delta}}
$$

is equal to the input SNR.

The absence of improvement of the signal-to-noise ratio in the linear amplification regime is explained by the fact that the coefficients of the Bogoliubov transformation are the same for the noise and for the signal. 


\section{Strong signal, four-mode noise squeezing}

The situation is different for the nonlinear amplification discussed in Sec. III C 3, where the nonlinear frequency shifts define the height and the width of the resonance, $\alpha_{n}\left|A_{n}\right|^{2} \gg \sqrt{\left|\Gamma_{1} \Gamma_{2}-\epsilon^{2}\right|}$. We remind the reader that this regime is valid across the threshold region, including the oscillator region, as long as the intracavity field of the signal dominates over the oscillation field.

In this case, the components of the noise spectral density are expressed through the matrix Bogoliubov coefficients in Eqs. (78) and (79), and they have the form

$$
\begin{aligned}
S_{n m}^{\theta}(\Delta)= & U_{n k}(\Delta) U_{m k}^{*}(\Delta)+V_{n k}^{*}(-\Delta) V_{m k}(-\Delta) \\
& +U_{n k}(\Delta) V_{m k}(-\Delta) e^{-2 i \theta} \\
& +V_{n k}^{*}(-\Delta) U_{m k}^{*}(\Delta) e^{2 i \theta}
\end{aligned}
$$

Because of the presence of the secondary idlers, one would expect that the noise of one mode could already be squeezed. Using the balanced-mode model, we compute the diagonal component, $S_{11}$, in Eq. (122),

$$
\begin{aligned}
S_{11}^{\theta}(\Delta)= & \frac{1}{2} \sum_{\sigma= \pm}\left(\left[\left|u_{\sigma}(\Delta)\right|-\left|v_{\sigma}(-\Delta)\right|\right]^{2}\right. \\
& \left.+2\left|u_{\sigma}(\Delta) v_{\sigma}(-\Delta)\right|\left\{1+\cos \left[\psi+\chi_{\sigma}(\Delta)-2 \theta\right]\right\}\right) .
\end{aligned}
$$

This component consists of independent contributions of both supermodes, where each contains an interference term similar to Eq. (112).

The optimum squeezing direction for each supermode is defined by the phase, $\chi_{\sigma}(\Delta)=\arg \left[u_{\sigma}(\Delta) v_{\sigma}(-\Delta)\right]$, and these phases are different for different supermodes, $\chi_{\sigma}(0)=-\sigma \pi / 2$, as follows from Eq. (82). Together with the value $\psi=2 \theta_{B}+\pi / 2$, which follows from Eq. (55), this gives the interference terms in Eq. (123) proportional to $\left[1+\sigma \cos 2\left(\theta_{B}-\theta\right)\right]$. Therefore, the two noise supermodes cannot be squeezed simultaneously.

The spectral power of the coherent signal has a form similar to Eq. (118), with the nonlinear gain [cf. Eqs. (52) and (48)]

$$
G_{11}(0) \approx\left(\frac{2 \Gamma^{2}}{\zeta^{2}}\right)^{2}
$$

and the maximum-amplification direction $\theta=\theta_{B}$. Comparing this value with the noise squeezing directions we find that it coincides with the squeezing direction of the supermode $\sigma=-$, while the contribution of the supermode $\sigma=+$ is amplified. The noise spectral density of the latter component has a sharp peak confined to the interval $|\Delta| \lesssim \varsigma_{+}^{2} / \Gamma$, according to Eq. (82), and has the peak value

$$
S_{11}^{\theta_{B}}(0) \approx 2\left(\frac{2 \Gamma^{2}}{3 \zeta^{2}}\right)^{2}=\frac{2}{9} G_{11}(0)
$$

The corresponding output SNR is,

$$
\mathrm{SNR}=36 \pi \frac{\left|B_{1}\right|^{2}}{\bar{\Delta}}
$$

which is 9 times larger than the linear result, Eq. (121). This enhancement of the signal-to-noise ratio results from the difference between the differential gain, which characterizes the noise, and the nonlinear gain of the signal; the former is always smaller for the nonlinear amplification.

A detailed numerical study confirms the validity of the adopted approximations for the absolute values of the intracavity fields and the Bogoliubov coefficients that lead to Eq. (126). However, the numerically evaluated phases of these quantities deviate from the analytical values, indicating high sensitivity of the phases to the approximation made. This deviation leads to drastic further enhancement of the SNR.

The result of the numerical study is illustrated in Fig. 12 for the representative case of the signal input, $\left|B_{1}\right|^{2}=0.1 \Gamma$, at the threshold, $\epsilon=\Gamma$. In Fig. 12(a), we show the noise spectral density $S_{11}^{\theta}(\Delta)$ versus $\Delta$ and $\theta$, and, in Fig. 12(b), we compare $S_{11}^{\theta}(0)$ (the red line) with the spectral power of the signal $\overline{P_{0}^{\theta}}$ (the green dashed line). As expected, the squeezing direction of the supermode $\sigma=-$ (the light-blue dotted line) approximately coincides with the maximum signal amplification. However, the spectrum of the supermode $\sigma=+$ (the dark-blue dotted line) is shifted by less than $\pi / 2$ from the spectrum of the supermode $\sigma=-$. This shift results in stronger suppression of the overall noise in the direction of the maximum signal amplification. The maximum SNR value is achieved at $\theta=\theta_{B}+0.06 \pi$, where $\overline{P_{0}} \approx 1820 \pi\left|B_{1}\right|^{2}$ and $S_{11}(0) \approx 14.5$, giving

$$
\mathrm{SNR} \approx 125 \pi \frac{\left|B_{1}\right|^{2}}{\bar{\Delta}}
$$

This is about 30 times larger than the linear result, and 15 times larger than the input value.

We conclude this section with a discussion of the noise squeezing in a homodyne detection with two local oscillators. To this end, we compute the cross-mode correlation function,

$$
\begin{aligned}
S_{12}^{\theta}(\Delta)= & \frac{1}{2} \sum_{\sigma= \pm} \sigma\left\{\left|u_{\sigma}(\Delta)\right|^{2} e^{i \psi}+\left|v_{\sigma}(-\Delta)\right|^{2} e^{-i \psi r}\right. \\
& \left.+\left[u_{\sigma}(\Delta) v_{\sigma}(-\Delta) e^{-2 i \theta}+\text { c.c. }\right]\right\} \\
= & {\left[S_{21}^{\theta}(\Delta)\right]^{*}, }
\end{aligned}
$$




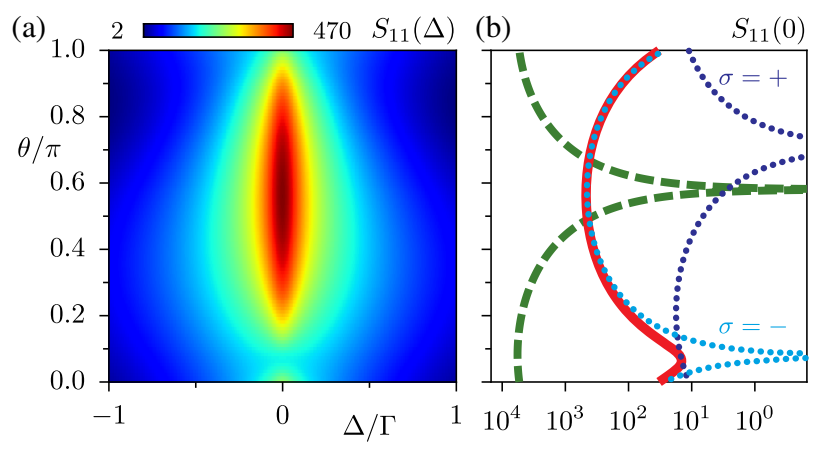

FIG. 12. Output noise squeezing in the presence of strong onresonance input $B_{1}$, mixed with a single local oscillator at frequency $\omega_{1}$ and phase $\theta$. (a) Noise squeezing spectrum $S_{11}^{\theta}(\Delta)$, Eq. (122), using linearized matrix Bogoliubov coefficients from Eqs. (66)-(69). (b) Noise $S_{11}^{\theta}(0)$ vs $\theta$ (the red solid line) and its decomposition in supermode contributions, Eq. (123) (the blue dashed line and the light-blue dotted line); the classical quadrature response $\overline{P_{0}} /\left|B_{1}\right|^{2}$, Eq. (106), with amplitude $C_{1}$ calculated from Eqs. (39)-(41) is indicated by the green dashed line. $\epsilon=\Gamma$, $\delta=0,\left|B_{1}\right|^{2}=0.1 \Gamma, \theta_{B}=0, \Gamma_{1}=\Gamma_{2}, \alpha_{1}=\alpha_{2}=\Gamma / 100$, and $\Gamma_{n}=\Gamma_{n 0}$.

and collect all of the correlation-function components to obtain after some algebra,

$$
\begin{aligned}
S^{\theta}(\Delta)= & 2\left\{e^{-2 r_{+}}+\sinh 2 r_{+}\left[1+\cos \left(\chi_{+}-2 \theta\right)\right]\right\} \cos ^{2} \frac{\psi}{2} \\
& +2\left\{e^{-2 r_{-}}+\sinh 2 r_{-}\left[1-\cos \left(\chi_{-}-2 \theta\right)\right]\right\} \sin ^{2} \frac{\psi}{2},
\end{aligned}
$$

here, $\left|v_{\sigma}(\Delta)\right|=\sinh r_{\sigma}(\Delta)$.

Given the relation $\chi_{\sigma}(0)=-\sigma \pi / 2$, we find that the optimum squeezing is achieved at $\theta=\pi / 4$ regardless of the $\psi$ value, where both terms in the square brackets turn to zero. Therefore, the full squeezing of the noise is possible. At the same time, the spectral power of the strong coherent signal in this case is given by Eq. (120), with $\chi(0)=-\pi / 2$, and the signal turns to zero for the noise squeezing direction $\theta=\pi / 4$.

A more accurate numerical computation of the nonlinear gain and the squeezing spectral density supports our conclusion based on analytics about coinciding directions of the signal and total noise squeezing, although numerical value for this direction deviates from the analytical one, $\theta \approx 0.36 \pi$, as illustrated in Fig. 13 .

\section{Squeezed vacuum}

In this section, we discuss the properties of the squeezedvacuum noise under nondegenerate parametric resonance.

In the case of two-mode squeezing, the Bogoliubov transformation, Eq. (56), can be written through a squeezing operator similar to the degenerate resonance [43],

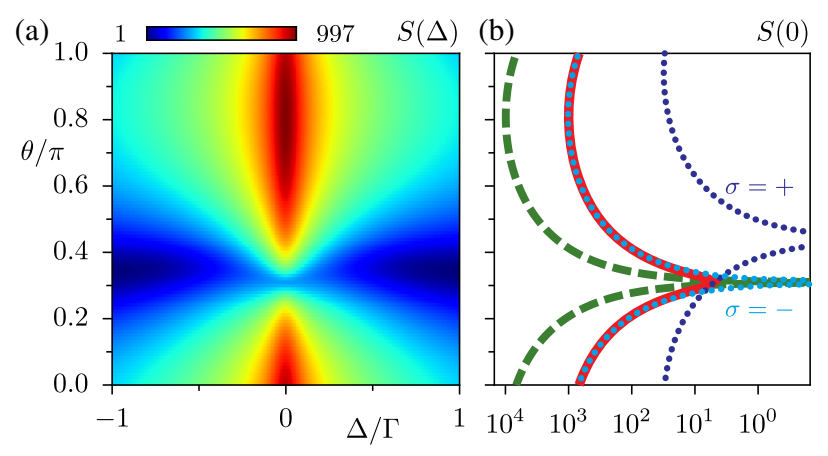

FIG. 13. Output noise in the presence of strong on-resonance input $B_{1}$, mixed with two local oscillators, at frequencies $\omega_{1}$ and $\omega_{2}$. (a) Noise squeezing spectrum $S^{\theta}(\Delta)$ from the sum over the components in Eq. (122). (b) Noise $S^{\theta}(0)$ vs $\theta$ (the red solid line) and the classical quadrature response $\overline{P_{0}} /\left|B_{1}\right|^{2}$, Eq. (106) (the green dashed line), the blue dashed and light-blue dotted lines indicate the supermode contributions. The parameters are as those used in Fig. 12.

$$
\begin{aligned}
c_{n}(\Delta) & =e^{i \eta_{n}(\Delta)} S b_{n}(\Delta) S^{\dagger}, \\
S & =\exp \left[\int_{-\infty}^{\infty} d \Delta^{\prime} \xi\left(\Delta^{\prime}\right) b_{1}^{\dagger}\left(\Delta^{\prime}\right) b_{2}^{\dagger}\left(-\Delta^{\prime}\right)-\text { H.c. }\right],
\end{aligned}
$$

where $\xi(\Delta)=r(\Delta) e^{i \rho(\Delta)}$, with $\rho=\arg (v / u)+\pi$, and $\eta_{n}=\arg u_{n}[\rho(\Delta)$ and $r(\Delta)$ are identical for both modes by virtue of the second property in Eq. (58)]. Then the squeezed-vacuum wave function has the form

$$
|\Psi\rangle=S|0\rangle,
$$

and it can be written explicitly using the decomposition formula $[44,45]$ and skipping the phase prefactor,

$$
|\Psi\rangle=\prod_{\Delta} \sum_{n=0}^{\infty} \frac{g^{n}(\Delta)}{\cosh r(\Delta)}|n(1, \Delta)\rangle|n(2,-\Delta)\rangle ;
$$

here, $g(\Delta)=\tanh r(\Delta) e^{i \rho(\Delta)}$, and $|n(j, \Delta)\rangle$ is the $n$-photon state at the frequency $\omega_{j}+\delta$ with detuning $\Delta$. In other words, the squeezed vacuum consists of an uncorrelated set of states, each being formed by correlated photon pairs of conjugated modes, $(1, \Delta),(2,-\Delta)$.

For the four-mode squeezing, we start with the Bogoliubov transformation in the supermode basis and present Eq. (75) in the form

$$
\begin{aligned}
c_{\sigma}(\Delta) & =S b_{\sigma}(\Delta) S^{\dagger}, \\
S & =\exp \left[\sum_{\sigma} \int_{0}^{\infty} d \Delta^{\prime} \xi_{\sigma}\left(\Delta^{\prime}\right) b_{\sigma}^{\dagger}\left(\Delta^{\prime}\right) b_{\sigma}^{\dagger}\left(-\Delta^{\prime}\right)-\text { H.c. }\right],
\end{aligned}
$$

where $\xi_{\sigma}(\Delta)=r_{\sigma}(\Delta) e^{i \rho_{\sigma}(\Delta)}$ and $\rho_{\sigma}=\arg \left(v_{\sigma} / u_{\sigma}\right)+\pi$, and where we omit a phase prefactor. Since the operator $S$ is a 
scalar in the mode space, the Bogoliubov transformation for the original modes can be written through the same operator,

$$
c_{n}(\Delta)=S b_{n}(\Delta) S^{\dagger},
$$

and the exponent of $S$ expressed in the original mode basis reads

$\sum_{\sigma, k l} \int_{0}^{\infty} d \Delta^{\prime} \xi_{\sigma}\left(\Delta^{\prime}\right) \mathfrak{U}_{\sigma k}^{T} \mathfrak{U}_{\sigma l}^{T} b_{k}^{\dagger}\left(\Delta^{\prime}\right) b_{l}^{\dagger}\left(-\Delta^{\prime}\right)-$ H.c.

The four-mode squeezed-vacuum wave function has the form

$$
\begin{aligned}
|\Psi\rangle= & \prod_{\Delta>0} \frac{1}{\cosh r_{+}(\Delta) \cosh r_{-}(\Delta)}, \\
& \times \exp \left\{\frac { g _ { + } + g _ { - } } { 2 } \left[e^{i \psi} b_{1}^{\dagger}(\Delta) b_{1}^{\dagger}(-\Delta)\right.\right. \\
& \left.\left.+e^{-i \psi} b_{2}^{\dagger}(\Delta) b_{2}^{\dagger}(-\Delta)\right]\right\}, \\
& \times \exp \left\{\frac{g_{+}-g_{-}}{2}\left[b_{1}^{\dagger}(\Delta) b_{2}^{\dagger}(-\Delta)+b_{2}^{\dagger}(\Delta) b_{1}^{\dagger}(-\Delta)\right]\right\}|0\rangle,
\end{aligned}
$$

with $g_{\sigma}=\tanh r_{\sigma} e^{i \rho_{\sigma}}$. This four-mode vacuum consists of a set of independent correlated photon pairs that belong to all possible combinations of the state quartet, $(1, \pm \Delta)$, $(2, \pm \Delta)$. It is worth noting that the admixture of the pairs from the same mode (the first of the two exponentials) is controlled by the intracavity field, $A_{n}$, and is sensitive to the phase of the strong field $\psi$, while the admixture of the pairs that belong to the different modes (the third line) is predominantly controlled by the flux pumping, $\epsilon$.

\section{FREQUENCY CONVERSION}

In this last section, we consider the regime of frequency conversion. In this regime, the pump frequency is chosen close to difference of the cavity resonances, $\Omega=\omega_{2}-$ $\omega_{1}+2 \delta, \omega_{2}>\omega_{1}$, and the dynamics of the system is described by Eq. (20) in the rotating frame $\omega_{1}-\delta$ and $\omega_{2}+\delta$.

Consider a nonlinear response to an input consisting of equally detuned signals in each mode, $B_{n}(t)=B_{n}(\Delta) e^{-i \Delta t}$. The classical dynamical equations for the intracavity-field amplitudes, $A_{n}(t)=A_{n}(\Delta) e^{-i \Delta t}$, read

$$
\begin{aligned}
& \left(\Delta+\zeta_{1}+i \Gamma_{1}\right) A_{1}(\Delta)+\epsilon A_{2}(\Delta)=\sqrt{2 \Gamma_{10}} B_{1}(\Delta), \\
& \left(\Delta+\zeta_{2}+i \Gamma_{2}\right) A_{2}(\Delta)+\epsilon A_{1}(\Delta)=\sqrt{2 \Gamma_{20}} B_{2}(\Delta) .
\end{aligned}
$$

Here, the $\zeta_{n}$ values are defined slightly differently compared to the amplification case,

$$
\begin{aligned}
& \zeta_{1}=-\delta+\alpha_{1}\left|A_{1}\right|^{2}+2 \alpha\left|A_{2}\right|^{2}, \\
& \zeta_{2}=\delta+\alpha_{2}\left|A_{2}\right|^{2}+2 \alpha\left|A_{1}\right|^{2} .
\end{aligned}
$$

Solving for $A_{n}$ we derive the input-output relation

$$
\left(\begin{array}{l}
C_{1} \\
C_{2}
\end{array}\right)=\mathcal{V}(\Delta)\left(\begin{array}{l}
B_{1} \\
B_{2}
\end{array}\right)
$$

where $\mathcal{V}(\Delta)$ is the intermode scattering matrix with the matrix elements

$$
\begin{aligned}
\mathcal{V}_{11}(\Delta) & =1-\frac{2 i \Gamma_{10}\left(\Delta+i \Gamma_{2}+\zeta_{2}\right)}{D(\Delta)}, \\
\mathcal{V}_{22}(\Delta) & =1-\frac{2 i \Gamma_{20}\left(\Delta+i \Gamma_{1}+\zeta_{1}\right)}{D(\Delta)}, \\
\mathcal{V}_{12}(\Delta) & =\frac{2 i \epsilon \sqrt{\Gamma_{10} \Gamma_{20}}}{D(\Delta)}=\mathcal{V}_{21}(\Delta), \\
D(\Delta) & =\left(\Delta+i \Gamma_{1}+\zeta_{1}\right)\left(\Delta+i \Gamma_{2}+\zeta_{2}\right)-\epsilon^{2} .
\end{aligned}
$$

In the absence of internal damping, $\Gamma_{n}=\Gamma_{n 0}$, this matrix is unitary,

$\mathcal{V} \mathcal{V}^{\dagger}=\mathcal{V}^{\dagger} \mathcal{V}=1, \quad\left|\mathcal{V}_{22}\right|=\left|\mathcal{V}_{11}\right|, \quad\left|\mathcal{V}_{12}\right|=\left|\mathcal{V}_{21}\right|$

which ensures preservation of the photon number during the conversion.

We note that the unitary property of the scattering matrix is to be considered with care since the transformation, Eqs. (139) and (140), is not a linear operation. It implicitly depends on the input via the intracavity Kerr effect, Eq. (138). Thus, this transformation cannot be automatically extended to the quantum regime.

The denominator in Eq. (140) never turns to zero; thus, no intrinsic instability occurs, and a sufficiently small input induces a small intracavity field. In this case, a linear approximation is appropriate, $\zeta_{1,2} \approx \mp \delta$, and Eqs. (139) and (140) apply to the quantum regime.

In Fig. 14, we show the linear reflection [Fig. 14(a)] and conversion [Fig. 14(b)] spectra, $\left|\mathcal{V}_{11}\left(\delta_{1}\right)\right|^{2}$ and $\left|\mathcal{V}_{12}\left(\delta_{1}\right)\right|^{2}$, of the parametric conversion process for the input signal, $B_{1}\left(\delta_{1}\right)$, versus the input and pump detunings.

For a pump detuned far away from the resonance, $|\delta| \gg \sqrt{\Gamma_{1} \Gamma_{2}}$, the spectrum is dominated by a loss resonance centered at $\delta_{1}=0$ (the green dashed line and the green curve). Close to the parametric resonance, $\delta<\sqrt{\Gamma_{1} \Gamma_{2}}$, the intermode coupling appears as an avoided crossing [the red and blue dashed lines and curves in Fig. 14(a)] that is accompanied by the emergence of the converted signal, Fig. 14(b). The points of maximum conversion are indicated by white markers. 

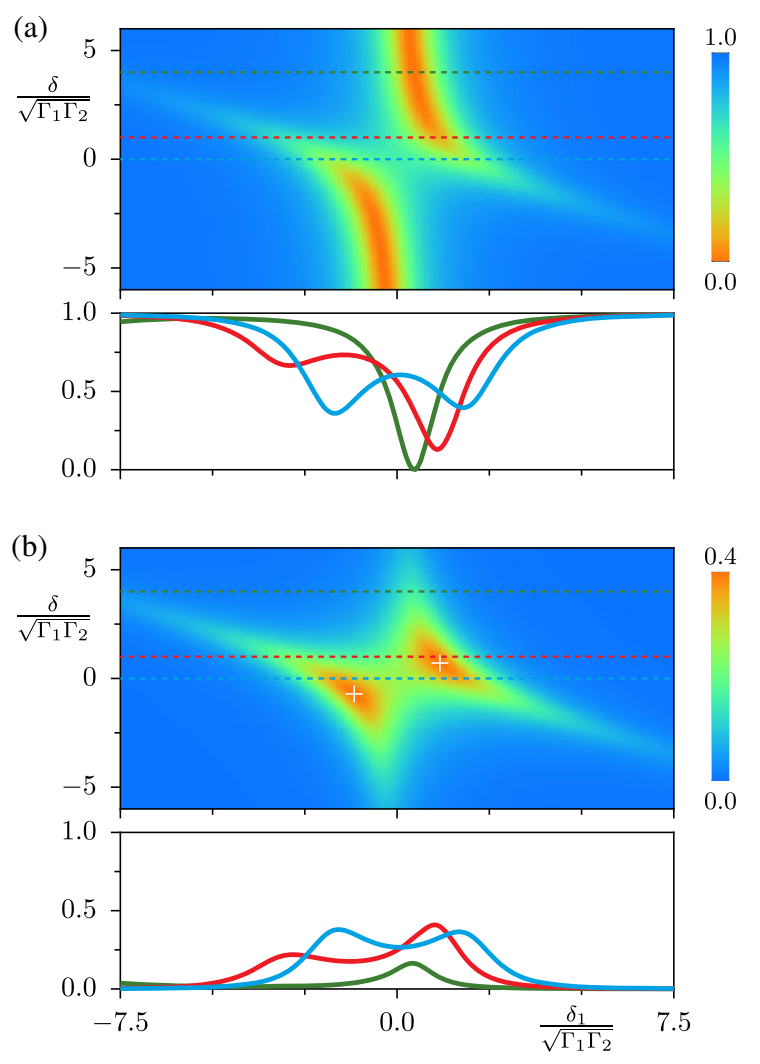

FIG. 14. Linear frequency conversion as function of inputsignal detuning $\delta_{1}$ and pump detuning $\delta$. (a) The reflection coefficient $\left|\mathcal{V}_{11}\left(\delta_{1}\right)\right|^{2}$ quantifies the response in the input mode and exhibits an avoided crossing of a loss resonance; the solid color lines in the lower panel correspond to the respective cuts at different $\delta$ 's in the upper panel, indicated by dashed lines. (b) The conversion coefficient $\left|\mathcal{V}_{12}\left(\delta_{1}\right)\right|^{2}$ quantifies the response in the second mode emerging at detuning, $\delta_{2}=2 \delta+\delta_{1}$; the white markers indicate points of maximum conversion. Observe different color codes in the main panels of (a) and (b). $\Gamma_{20}=3 \Gamma_{10}, \Gamma_{1}=1.8 \Gamma_{10}, \Gamma_{2}=4 \Gamma_{10}=4 / 3 \Gamma_{20}, \alpha_{2}=3 \alpha_{1}$, and $\alpha_{1}=\sqrt{\Gamma_{10} \Gamma_{20}} / 100$.

A full reciprocal conversion between the modes is possible in the absence of internal losses. The criterion is given by the zero reflection coefficient, $\left|\mathcal{V}_{11}\right|^{2}=0$. The corresponding conditions read, for the linear regime,

$\epsilon^{2}=\Gamma_{1} \Gamma_{2}\left(1+\frac{4 \delta^{2}}{\left(\Gamma_{2}-\Gamma_{1}\right)^{2}}\right), \quad \Delta=\frac{\Gamma_{1}+\Gamma_{2}}{\Gamma_{2}-\Gamma_{1}} \delta$

It is instructive to compare these equations to the ones for the parametric instability in the amplification regime, Eqs. (27) and (28): the two criteria coincide at the zero pump detuning, $\delta=0$. At finite pump detuning, full conversion is still possible, but, in this case, the input must be detuned accordingly. The efficiency of the frequency conversion at different pump strengths is illustrated in Fig. 15.

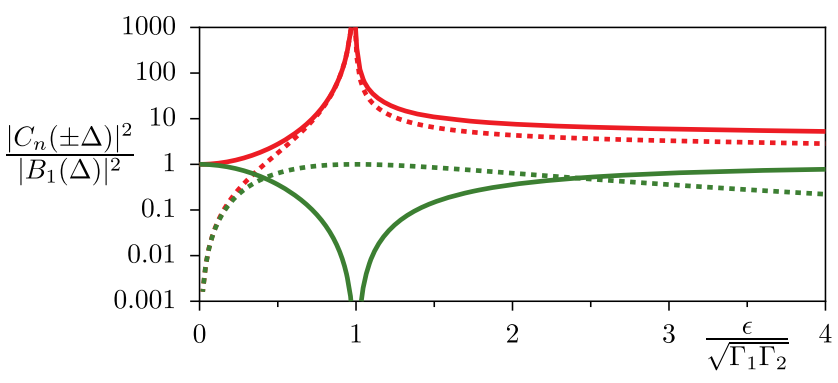

FIG. 15. Efficiency of the (linear) parametric conversion vs the pump strength $\epsilon$ (the green lines), characterized by the conversion (dashed line) and reflection (solid line) coefficients. These coefficients are compared with the gains in the parametric amplification (the red lines) of a signal (the solid line) and an idler (the dashed line). $\delta=0, \delta_{1}=0, \Gamma_{2}=3 \Gamma_{1}$, and $\alpha_{2}=3 \alpha_{1}=$ $3 \sqrt{\Gamma_{1} \Gamma_{2}} / 100$.

\section{CONCLUSION}

In this paper, we study nondegenerate parametric resonance in a tunable superconducting microwave cavity. The main focus is put on the nonlinear properties of the resonance, stemming from the nonlinear current-phase dependence of the SQUID controlling the cavity. We analyze nonlinear gains in the strong amplification regime at the parametric-oscillation threshold, and we evaluate the maximum values of the gains. We show that the linear response of an empty cavity has the property of two-mode squeezing, while the response of a cavity filled with radiation has a four-mode structure. We identify the parametric-oscillation regime and show that the oscillation frequencies deviate from the cavity resonances, with the deviations growing with the strength of the pump. A continuous degeneracy of the oscillator state with respect to the oscillation phase causes divergence of the linear response at the oscillation frequencies for all pump strengths above the threshold. We find that injection of a weak onresonance signal locks the oscillation phase and makes the response regular. We also calculate noise squeezing spectral densities in the two-mode and four-mode regimes, and we find that the output signal-to-noise ratio in the four-mode regime can significantly exceed the input value. Finally, we investigate the parametric frequency conversion and identify the conditions for full and reversible conversion.

\section{ACKNOWLEDGMENTS}

The authors are grateful to Andreas Bengtsson, Per Delsing, and Giulia Ferrini for the useful discussions. Support from the Knut and Alice Wallenberg Foundation is gratefully acknowledged.

\section{APPENDIX: PARAMETRIC-OSCILLATION STATE}

To find solutions of Eq. (24) for a self-sustained oscillation above the parametric threshold, we consider 
the ansatz $A_{1,2}(t)=r_{1,2} e^{i \theta_{1,2}} e^{\mp i \Delta_{0} t}$ and substitute it into the homogeneous equation

$$
\begin{aligned}
\left(\Delta_{0}+\zeta_{1}+i \Gamma_{1}\right) r_{1}+\epsilon r_{2} e^{-i \Theta} & =0, \\
\left(-\Delta_{0}+\zeta_{2}+i \Gamma_{2}\right) r_{2}+\epsilon r_{1} e^{-i \Theta} & =0,
\end{aligned}
$$

where $\Theta=\theta_{1}+\theta_{2}$. Then we separate the real and imaginary parts of the equations.

The imaginary parts read

$$
\begin{aligned}
& \Gamma_{1} r_{1}-\epsilon r_{2} \sin \Theta=0, \\
& \Gamma_{2} r_{2}-\epsilon r_{1} \sin \Theta=0
\end{aligned}
$$

and yield

$$
\frac{r_{1}}{r_{2}}=\sqrt{\frac{\Gamma_{2}}{\Gamma_{1}}}, \quad \sin \Theta=\frac{\sqrt{\Gamma_{1} \Gamma_{2}}}{\epsilon}>0 .
$$

The real parts read

$$
\begin{aligned}
\left(\Delta_{0}+\zeta_{1}\right) r_{1}+\epsilon r_{2} \cos \Theta & =0 \\
\left(-\Delta_{0}+\zeta_{2}\right) r_{2}+\epsilon r_{1} \cos \Theta & =0
\end{aligned}
$$

from which we extract the relation

$$
\frac{\Delta_{0}+\zeta_{1}}{-\Delta_{0}+\zeta_{2}}=\frac{r_{2}^{2}}{r_{1}^{2}}=\frac{\Gamma_{1}}{\Gamma_{2}},
$$

which defines the detuning $\Delta_{0}$,

$$
\Delta_{0}=\frac{\zeta_{2} \Gamma_{1}-\zeta_{1} \Gamma_{2}}{\Gamma_{1}+\Gamma_{2}}
$$

Using this equation, we compute

$$
\Delta_{0}+\zeta_{1}=\frac{\left(\zeta_{1}+\zeta_{2}\right) \Gamma_{1}}{\Gamma_{1}+\Gamma_{2}}
$$

and, substituting in Eq. (A4), we derive an equation for $\cos \Theta$,

$$
\cos \Theta=-\frac{\zeta_{1}+\zeta_{2}}{\epsilon} \frac{\sqrt{\Gamma_{1} \Gamma_{2}}}{\Gamma_{1}+\Gamma_{2}}= \pm \frac{\sqrt{\epsilon-\Gamma_{1} \Gamma_{2}}}{\epsilon} .
$$

Writing explicitly $\zeta_{n}$ through $r_{n}$ in this equation and excluding $r_{2}$ using Eq. (A3), we finally get

$$
r_{1}^{2}=\frac{2\left(-\delta \mp \delta_{\mathrm{th}}\right) \Gamma_{2}}{\alpha_{1} \Gamma_{2}+\alpha_{1} \Gamma_{2}+2 \alpha\left(\Gamma_{1}+\Gamma_{2}\right)},
$$

where we also use Eq. (29). The upper (lower) sign in Eqs. (A8) and (A9) corresponds to the unstable (stable) solution.
[1] T. Yamamoto, K. Inomata, M. Watanabe, K. Matsuba, T. Miyazaki, W. D. Oliver, Y. Nakamura, and J.S. Tsai, Flux-driven Josephson parametric amplifier, Appl. Phys. Lett. 93, 042510 (2008).

[2] M. A. Castellanos-Beltran and K. W. Lehnert, Widely tunable parametric amplifier based on a superconducting quantum interference device array resonator, Appl. Phys. Lett. 91, 083509 (2007).

[3] M. A. Castellanos-Beltran, K. D. Irwin, G. C. Hilton, L. R. Vale, and K.W. Lehnert, Amplification and squeezing of quantum noise with a tunable Josephson metamaterial, Nat. Phys. 4, 929 (2008).

[4] N. Bergeal, F. Schackert, M. Metcalfe, R. Vijay, V.E. Manucharyan, L. Frunzio, D. E. Prober, R. J. Schoelkopf, S. M. Girvin, and M. H. Devoret, Phase-preserving amplification near the quantum limit with a Josephson ring modulator, Nature (London) 465, 64 (2010).

[5] J. Mutus et al., Design and characterization of a lumped element single-ended superconducting microwave parametric amplifier with on-chip flux bias line, Appl. Phys. Lett. 103, 122602 (2013).

[6] X. Zhou, V. Schmitt, P. Bertet, D. Vion, W. Wustmann, V. Shumeiko, and D. Esteve, High-gain weakly nonlinear flux-modulated Josephson parametric amplifier using a SQUID array, Phys. Rev. B 89, 214517 (2014).

[7] M. Hatridge, R. Vijay, D. H. Slichter, J. Clarke, and I. Siddiqi, Dispersive magnetometry with a quantum limited SQUID parametric amplifier, Phys. Rev. B 83, 134501 (2011).

[8] C. Macklin, K. O. Brien, D. Hover, M. E. Schwartz, V. Bolkhovsky, X. Zhang, W. D. Oliver, and I. Siddiqi, A near-quantum-limited Josephson traveling-wave parametric amplifier, Science 350, 307 (2015).

[9] M. Simoen, C. W. S. Chang, P. Krantz, J. Bylander, W. Wustmann, V. Shumeiko, P. Delsing, and C. M. Wilson, Characterization of a multimode coplanar waveguide parametric amplifier, J. Appl. Phys. 118, 154501 (2015).

[10] R. Vijay, D. H. Slichter, and I. Siddiqi, Observation of Quantum Jumps in a Superconducting Artificial Atom, Phys. Rev. Lett. 106, 110502 (2011).

[11] K. W. Murch, S. J. Weber, C. Macklin, and I. Siddiqi, Observing single quantum trajectories of a superconducting quantum bit, Nature (London) 502, 211 (2013).

[12] Z. R. Lin, K. Inomata, W. D. Oliver, K. Koshino, Y. Nakamura, J. S. Tsai, and T. Yamamoto, Single-shot readout of a superconducting flux qubit with a flux-driven Josephson parametric amplifier, Appl. Phys. Lett. 103, 132602 (2013).

[13] D. Riste, M. Dukalski, C. A. Watson, G. de Lange, M. J. Tiggelman, Y. M. Blanter, K. W. Lehnert, R. N. Schouten, and L. DiCarlo, Deterministic entanglement of superconducting qubits by parity measurement and feedback, Nature (London) 502, 350 (2013).

[14] P. Krantz, A. Bengtsson, M. Simoen, S. Gustavsson, V. Shumeiko, W. D. Oliver, C. M. Wilson, P. Delsing, and J. Bylander, Single-shot read-out of a superconducting qubit using a Josephson parametric oscillator, Nat. Commun. 7, 11417 (2016).

[15] C. Eichler, D. Bozyigit, C. Lang, M. Baur, L. Steffen, J. M. Fink, S. Filipp, and A. Wallraff, Observation of Two-Mode 
Squeezing in the Microwave Frequency Domain, Phys. Rev. Lett. 107, 113601 (2011).

[16] K. W. Murch, S. J. Weber, K. M. Beck, E. Ginossar, and I. Siddiqi, Reduction of the radiative decay of atomic coherence in squeezed vacuum, Nature (London) 499, 62 (2013).

[17] D. M. Toyli, A. W. Eddins, S. Boutin, S. Puri, D. Hover, V. Bolkhovsky, W. D. Oliver, A. Blais, and I. Siddiqi, Resonance Fluorescence from an Artificial Atom in Squeezed Vacuum, Phys. Rev. X 6, 031004 (2016).

[18] N. Bergeal, F. Schackert, L. Frunzio, and M. H. Devoret, Two-Mode Correlation of Microwave Quantum Noise Generated by Parametric Down-Conversion, Phys. Rev. Lett. 108, 123902 (2012).

[19] E. Flurin, N. Roch, F. Mallet, M. H. Devoret, and B. Huard, Generating Entangled Microwave Radiation over Two Transmission Lines, Phys. Rev. Lett. 109, 183901 (2012).

[20] N. Roch, E. Flurin, F. Nguyen, P. Morfin, P. CampagneIbarcq, M. H. Devoret, and B. Huard, Widely Tunable, Nondegenerate Three-Wave Mixing Microwave Device Operating near the Quantum Limit, Phys. Rev. Lett. 108, 147701 (2012).

[21] C. Eichler, C. Lang, J. M. Fink, J. Govenius, S. Filipp, and A. Wallraff, Observation of Entanglement Between Itinerant Microwave Photons and a Superconducting Qubit, Phys. Rev. Lett. 109, 240501 (2012).

[22] E. P. Menzel, R. Di Candia, F. Deppe, P. Eder, L. Zhong, M. Ihmig, M. Haeberlein, A. Baust, E. Hoffmann, D. Ballester, K. Inomata, T. Yamamoto, Y. Nakamura, E. Solano, A. Marx, and R. Gross, Path Entanglement of ContinuousVariable Quantum Microwaves, Phys. Rev. Lett. 109, 250502 (2012).

[23] E. Zakka-Bajjani, F. Nguyen, M. Lee, L. R. Vale, R. W. Simmonds, and J. Aumentado, Quantum superposition of a single microwave photon in two different "colour" states, Nat. Phys. 7, 599 (2011).

[24] B. Abdo, K. Sliwa, F. Schackert, N. Bergeal, M. Hatridge, L. Frunzio, A. D. Stone, and M. Devoret, Full Coherent Frequency Conversion between Two Propagating Microwave Modes, Phys. Rev. Lett. 110, 173902 (2013).

[25] W. Wustmann and V. Shumeiko, Parametric resonance in tunable superconducting cavities, Phys. Rev. B 87, 184501 (2013).

[26] M. Sandberg, C. M. Wilson, F. Persson, T. Bauch, G. Johansson, V. Shumeiko, T. Duty, and P. Delsing, Tuning the field in a microwave resonator faster than the photon lifetime, Appl. Phys. Lett. 92, 203501 (2008).

[27] M. Wallquist, V. S. Shumeiko, and G. Wendin, Selective coupling of superconducting charge qubits mediated by a tunable stripline cavity, Phys. Rev. B 74, 224506 (2006).
[28] C. M. Wilson, G. Johansson, A. Pourkabirian, M. Simoen, J. R. Johansson, T. Duty, F. Nori, and P. Delsing, Observation of the dynamical Casimir effect in a superconducting circuit, Nature (London) 479, 376 (2011).

[29] R. Graham and H. Haken, The quantum-fluctuations of the optical parametric oscillator, Z. Phys. 210, 276 (1968).

[30] D. F. Walls and G. J. Milburn, Quantum Optics (Springer, Berlin, 2008).

[31] C. M. Caves, Quantum limits on noise in linear amplifiers, Phys. Rev. D 26, 1817 (1982).

[32] M. O. Scully and M.S. Zubairy, Quantum Optics (Cambridge University Press, Cambridge, England, 2006).

[33] A. A. Clerk, M. H. Devoret, S. M. Girvin, F. Marquardt, and R. J. Schoelkopf, Introduction to quantum noise, measurement, and amplification, Rev. Mod. Phys. 82, 1155 (2010).

[34] C. Fabre, E. Giacobino, A. Heidmann, and S. Reynaud, Noise characteristics of a non-degenerate optical parametric oscillator-Application to quantum noise reduction, J. Phys. (Paris) 50, 1209 (1989).

[35] G. Björk and Y. Yamamoto, Generation and amplification of number states by nondegenerate parametric oscillators with idler-measurement feedback, Phys. Rev. A 37, 125 (1988).

[36] M. D. Reid and P. D. Drummond, Correlations in nondegenerate parametric oscillation: Squeezing in the presence of phase diffusion, Phys. Rev. A 40, 4493 (1989).

[37] A. S. Villar, M. Martinelli, and P. Nussenzveig, Testing the entanglement of intense beams produced by a nondegenerate optical parametric oscillator, Opt. Commun. 242, 551 (2004).

[38] C. Navarrete-Benlloch, A. Romanelli, E. Roldán, and G. J. de Valcárcel, Noncritical quadrature squeezing in two-transverse-mode optical parametric oscillators, Phys. Rev. A 81, 043829 (2010).

[39] S. L. Braunstein, Squeezing as an irreducible resource, Phys. Rev. A 71, 055801 (2005).

[40] G. Patera, N. Treps, C. Fabre, and G. J. de Valcarcel, Quantum theory of synchronously pumped type I optical parametric oscillators: Characterization of the squeezed supermodes, Eur. Phys. J. D 56, 123 (2010).

[41] M. J. Collett and C. W. Gardiner, Squeezing of intracavity and traveling-wave light fields, produced in parametric amplification, Phys. Rev. A 30, 1386 (1984).

[42] B. Yurke, in Quantum Squeezing, edited by P.D. Drummond and Z. Fizek (Springer, Berlin, 2004).

[43] P. D. Drummond, in Quantum Squeezing, edited by P. D. Drummond and Z. Fizek (Springer, Berlin, 2004).

[44] M. J. Collett, Exact density-matrix calculations for simple open systems, Phys. Rev. A 38, 2233 (1988).

[45] A. M. Perelomov, Generalized coherent states and some of their applications, Sov. Phys. Usp. 20, 703 (1977). 